\title{
ABUNDANCES, STELLAR PARAMETERS, AND SPECTRA FROM THE SDSS-III/APOGEE SURVEY
}

\author{
Jon A. Holtzman ${ }^{1}$, Matthew Shetrone ${ }^{2}$, Jennifer A. Johnson ${ }^{3}$, Carlos Allende Prieto ${ }^{4,5}$, Friedrich Anders ${ }^{6,7}$, \\ Brett Andrews ${ }^{8}$, Timothy C. Beers ${ }^{9}$, Dmitry Bizyaev ${ }^{10}$, Michael R. Blanton ${ }^{11}$, Jo Bovy ${ }^{12,30}$, Ricardo Carrera ${ }^{4,5}$, \\ S. Drew Chojnowski ${ }^{1}$, Katia Cunha ${ }^{13,14}$, Daniel J. Eisenstein ${ }^{15,16}$, Diane Feuillet ${ }^{1}$, Peter M. Frinchaboy ${ }^{15}$, \\ Jessica Galbraith-Frew ${ }^{17}$, Ana E. García Pérez ${ }^{4,5}$, D. A. García-Hernández ${ }^{4,5}$, Sten Hasselquist ${ }^{1}$, Michael R. Hayden ${ }^{1}$, \\ Fred R. Hearty ${ }^{18,19}$, Inese Ivans ${ }^{17}$, Steven R. Majewski ${ }^{20}$, Sarah Martell ${ }^{21}$, Szabolcs Meszaros ${ }^{22}$, Demitri Muna ${ }^{3}$, \\ David Nidever $^{23}$, Duy Cuong Nguyen ${ }^{24}$, Robert W. O'Connell ${ }^{20}$, Kaike Pan ${ }^{10}$, Marc Pinsonneault ${ }^{3}$, Annie C. Robin ${ }^{25}$, \\ Ricardo P. Schiavon ${ }^{26,27}$, Neville Shane ${ }^{20}$, Jennifer Sobeck ${ }^{20}$, Verne V. Smith ${ }^{28}$, Nicholas Troup ${ }^{20}$, David H. Weinberg ${ }^{3}$, \\ John C. Wilson ${ }^{20}$, W. M. WoOd-VASEY ${ }^{8}$, Olga Zamora ${ }^{4,5}$, and GaIL Zasowski ${ }^{29}$ \\ ${ }^{1}$ New Mexico State University, Las Cruces, NM 88003, USA; holtz@nmsu.edu, mrhayden@nmsu.edu, feuilldk@nmsu.edu \\ ${ }^{2}$ University of Texas at Austin, McDonald Observatory, Fort Davis, TX 79734, USA; shetrone@astro.as.utexas.edu \\ ${ }^{3}$ Department of Astronomy, The Ohio State University, Columbus, OH 43210, USA; jaj@astronomy.ohio-state.edu, muna@astronomy.ohio-state.edu \\ ${ }^{4}$ Instituto de Astrofísica de Canarias, E-38205 La Laguna, Tenerife, Spain \\ ${ }^{5}$ Departamento de Astrofísica, Universidad de La Laguna, E-38206 La Laguna, Tenerife, Spain; callende@iac.es, agp@iac.es \\ 6 Leibniz-Institut fur Astrophysik Potsdam (AIP), An der Sternwarte 16, D-14482, Potsdam, Germany
${ }^{6}$, \\ ${ }^{7}$ Laboratório Interinstitucional de e-Astronomía (LIneA), Rua Gal. José Cristino 77, Rio de Janeíro, RJ-20921400, Brasil \\ ${ }^{8}$ PITT PACC, Department of Physics and Astronomy, University of Pittsburgh, Pittsburgh, PA 15260, USA; wmwv@ @itt.edu \\ ${ }^{9}$ Dept. of Physics and JINA Center for Evolution of the Elements, University of Notre Dame, Notre Dame, IN 46556, USA \\ ${ }^{10}$ Apache Point Observatory, P.O. Box 59, Sunspot, NM 88349-0059, USA; dmbiz@apo.nmsu.edu \\ Center for Cosmology and Particle Physics, Department of Physics, New York University, \\ 4 Washington Place, New York, NY 10003, USA; michael.blanton@gmail.com \\ 12 Institute for Advanced Study, Einstein Drive, Princeton, NJ 08540, USA; bovy@ias.edu \\ ${ }^{13}$ Observatório Nacional, São Cristóvão, Rio de Janeiro, Brazil \\ ${ }^{14}$ University of Arizona, Tucson, AZ 85719, USA; cunha@email.noao.edu \\ ${ }^{15}$ Texas Christian University, Fort Worth, TX 76129, USA; p.frinchaboy@ @ tcu.edu \\ ${ }^{16}$ Harvard-Smithsonian Center for Astrophysics, 60 Garden St., Cambridge, MA 02138, USA; deisenstein@cfa.harvard.edu \\ ${ }^{17}$ Physics and Astronomy, University of Utah, Salt Lake City, UT 84112, USA; iii@ physics.utah.edu \\ ${ }^{18}$ Department of Astronomy and Astrophysics, The Pennsylvania State University, University Park, PA 16802, USA; frh10@psu.edu \\ ${ }^{19}$ Institute for Gravitation and the Cosmos, The Pennsylvania State University, University Park, PA 16802, USA \\ ${ }^{20}$ Dept. of Astronomy, University of Virginia, Charlottesville, VA 22904-4325, USA; srm4n@ virginia.edu, aeg4x@ @irginia.edu, rwo@ virginia.edu \\ ${ }^{21}$ School of Physics, University of New South Wales, Sydney NSW 2 052, Australia \\ ${ }^{22}$ ELTE Gothard Astrophysical Observatory, H-9704 Szombathely, Szent Imre herceg st. 112, Hungary \\ ${ }^{23}$ Department of Astronomy, University of Michigan, Ann Arbor, MI 48109, USA; dnidever@umich.edu \\ ${ }^{24}$ Dunlap Institute for Astronomy and Astrophysics, University of Toronto, Toronto, Ontario, Canada \\ 25 Institut UTINAM, OSU THETA, University of Franche-Comté, Besançon, France; annie@ obs-besancon.fr \\ ${ }^{26}$ Gemini Observatory, 670 N A’Ohoku Place, Hilo, HI 96720, USA \\ 27 Astrophysics Research Institute, Liverpool John Moores University, Liverpool, L3 5RF, UK; rpschiavon@ gmail.com \\ ${ }^{28}$ National Optical Astronomy Observatories, Tucson, AZ 85719, USA; vsmith@email.noao.edu \\ ${ }^{29}$ Department of Physics and Astronomy, Johns Hopkins University, Baltimore, MD 21218, USA; gail.zasowski@gmail.com \\ Received 2015 January 16; accepted 2015 August 20; published 2015 October 14
}

\begin{abstract}
The SDSS-III/Apache Point Observatory Galactic Evolution Experiment (APOGEE) survey operated from 2011-2014 using the APOGEE spectrograph, which collects high-resolution $(R \sim 22,500)$, near-IR $(1.51-1.70 \mu \mathrm{m})$ spectra with a multiplexing (300 fiber-fed objects) capability. We describe the survey data products that are publicly available, which include catalogs with radial velocity, stellar parameters, and 15 elemental abundances for over 150,000 stars, as well as the more than 500,000 spectra from which these quantities are derived. Calibration relations for the stellar parameters $\left(T_{\text {eff }}, \log g,[\mathrm{M} / \mathrm{H}],[\alpha / \mathrm{M}]\right)$ and abundances (C, N, O, Na, Mg, Al, Si, S, K, Ca, Ti, V, Mn, Fe, Ni) are presented and discussed. The internal scatter of the abundances within clusters indicates that abundance precision is generally between 0.05 and 0.09 dex across a broad temperature range; it is smaller for some elemental abundances within more limited ranges and at high signal-to-noise ratio. We assess the accuracy of the abundances using comparison of mean cluster metallicities with literature values, APOGEE observations of the solar spectrum and of Arcturus, comparison of individual star abundances with other measurements, and consideration of the locus of derived parameters and abundances of the entire sample, and find that it is challenging to determine the absolute abundance scale; external accuracy may be good to 0.1-0.2 dex. Uncertainties may be larger at cooler temperatures $\left(T_{\text {eff }}<4000 \mathrm{~K}\right)$. Access to the public data release and data products is described, and some guidance for using the data products is provided.
\end{abstract}

Key words: astronomical databases: miscellaneous - Galaxy: abundances - stars: abundances - surveys

30 John Bahcall Fellow. 


\section{INTRODUCTION}

The third phase of the Sloan Digital Sky Survey (SDSS-III) included the Apache Point Observatory Galactic Evolution Experiment (APOGEE) as one of its key components. APOGEE made use of a new, multi-object, near-IR spectrograph to obtain spectra of more than 150,000 stars across portions of the Milky Way visible from the Apache Point Observatory (APO). The main goal of the APOGEE survey was to obtain high-resolution spectra to map out the kinematical and chemical structure of the Milky Way.

The overall design and goals of the SDSS-III survey are described in Eisenstein et al. (2011) and of APOGEE in S. R. Majewski et al. (2015, in preparation). The survey had a period of commissioning in spring 2011 and operated in survey-mode from fall 2011 until summer 2014. Data from the first year of operation was released as part of the SDSS DR10 (Ahn et al. 2014) and included measurements of basic stellar parameters as well as overall metal and $\alpha$-element abundances. An SDSS-III internal data release (DR11) included the first two years of survey data with the same analysis as was used for DR10. The final SDSS-III data release (DR12; Alam et al. 2015) includes the full 3 years of data from the SDSSIII/APOGEE survey. For DR12, all of the data have been reprocessed, and the parameter and abundance analysis have been significantly updated: APOGEE DR12 includes measurements of individual chemical abundances for 15 different elements.

The operation of the APOGEE instrument will continue in the APOGEE-2 survey, which is part of the fourth phase of the Sloan Digital Sky Survey (SDSS-IV) that plans to operate from 2014 to 2020. APOGEE-2 will consist of additional observations in the northern hemisphere from APO but will also include observations of the southern Milky Way that will be obtained with the $2.5 \mathrm{~m}$ du Pont telescope at Las Campanas Observatory. Future data releases for this survey may include reprocessing of APOGEE-1 data.

This paper provides a general overview of the data provided by the SDSS-III/APOGEE survey. Section 2 briefly summarizes the survey. Sections 3 and 4 summarize how the reduction and spectroscopic analysis is performed (but these are described in greater detail in Nidever et al. 2015 and A. E. García Pérez et al. 2015, in preparation) and introduce the reader to the associated data products. Section 5 discusses the calibration and validation of the derived stellar parameters and abundances. Options for accessing the data are presented in Section 6. Section 7 describes the APOGEE catalog data and presents some important considerations for users of these data, and Section 8 has a similar presentation for the spectra.

\section{THE SDSS-III/APOGEE SURVEY}

\subsection{The APOGEE Instrument}

The APOGEE survey utilizes a fiber spectrograph that records 300 spectra simultaneously. The spectrograph uses a volume phase holographic grating and camera optics to deliver spectra in the $\mathrm{H}$ band between 1.51 and $1.70 \mu \mathrm{m}$ at a spectral resolution of $R \sim 22,500$. The spectra are recorded on three Hawaii-2 RG detectors with small gaps in spectral coverage between the detectors. The instrument was constructed largely at the University of Virginia and is described in detail in J. Wilson et al. (2015, in preparation).
The APOGEE spectrograph was shipped to APO in the spring of 2011 and was commissioned during the period AprilJune of 2011. During the commissioning period, it was recognized that there was some astigmatism within the instrument, so modifications were made during summer 2011 to address this. Because of the optical issues and modifications after commissioning, the commissioning data have lower spectral resolution that does not meet the strict survey requirements, especially in the detector covering the longest wavelengths. Commissioning data have been released in the APOGEE data releases as these data provide useful radial velocity information, but abundance analysis is not currently provided for them because of the reduced resolution and quality. However, many of the sources observed during commissioning were reobserved during the main survey as time and weather permitted.

After the summer 2011 modifications, the instrument was pumped down and cooled in 2011 August and remained unopened, operating continuously without any large changes in pressure or temperature, for the duration of the SDSS-III/ APOGEE survey. Over the period of the main survey, the instrument met survey requirements and was remarkably stable, with essentially no changes in optical and detector performance.

At the shorter end of the wavelength range, the detector pixels do not quite adequately sample the spectrograph pointsource function. To ensure adequate spectral sampling, the instrument includes a dither mechanism for moving the entire detector array, and exposures are taken in pairs, with the detectors shifted 0.5 pixels between the two observations in a pair.

One of the detectors (at the short wavelength end) used in the SDSS-III/APOGEE survey exhibits strong persistence, where a significant fraction of accumulated charge is released over a long period of time (Nidever et al. 2015). This affects roughly a third of the chip and is distributed in such a way that about a third of the fibers are affected at all wavelengths on that chip $(1.51-1.58 \mu \mathrm{m})$, while the other two-thirds of the fibers are unaffected. The behavior of the persistence is challenging to characterize, and its relevance depends both on the brightness of a spectrum as well as the history of previous exposures. The current reduction analysis does not attempt to correct for this; we present some information on the impact on derived stellar parameters and abundances in Section 5.7. The offending detector was swapped out in summer 2014 after the completion of the SDSS-III/APOGEE survey.

\subsection{Survey Operations}

The primary source of the light to the spectrograph is the SDSS $2.5 \mathrm{~m}$ telescope (Gunn et al. 2006), which uses a plugplate system to collect light from a circular $3^{\circ}$ diameter field. For the vast majority of the observations, 230 of the fibers were placed on science targets, 35 fibers on hot star targets used for correction of telluric absorption (although these also enable some science projects), and 35 fibers on "blank" sky regions to enable sky subtraction.

Standard exposure sequences consist of 8 separate $500 \mathrm{~s}$ exposures, with 4 at each of the two dither positions, in an ABBAABBA pattern. The $4000 \mathrm{~s}$ integration time yields a signal-to-noise ratio $(\mathrm{S} / \mathrm{N})$ of about 100 per half-resolution element (a typical pixel) on a source with $H=11$, although the 

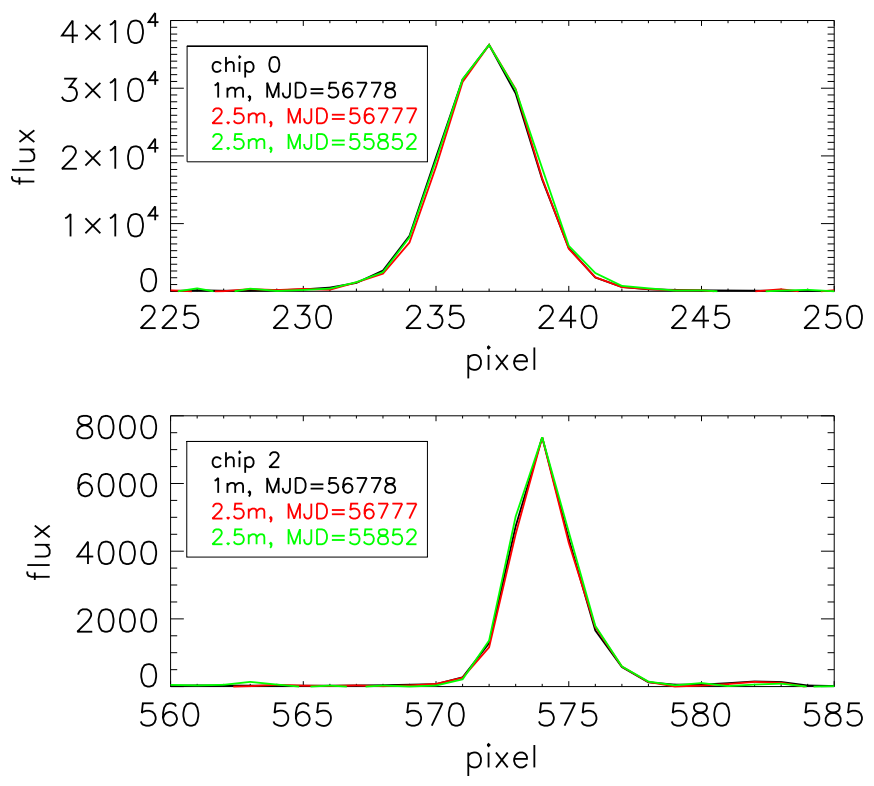

Figure 1. Comparison of sky emission lines as taken with the $2.5 \mathrm{~m}$ and $1 \mathrm{~m}$ telescopes.

sensitivity varies across the spectral region recorded on the detectors, primarily as a result of grating sensitivity changes.

\subsection{1 m Telescope Fiber Feed}

Partway through the survey, a fiber feed was constructed from the nearby NMSU $1 \mathrm{~m}$ telescope for 10 fibers, which are located in a fixed linear pattern in the focal plane of that telescope. This configuration enables observations of single objects, with accompanying sky spectra, and has been used to provide a sample of mostly bright stars, useful for both calibration and science, as discussed in Section 5.5. To use the $1 \mathrm{~m}$ observations to validate those obtained with the $2.5 \mathrm{~m}$, the spectra from the two telescopes must match closely. The input beam from the $2.5 \mathrm{~m}$ telescope is $f / 5$, while that of the $1 \mathrm{~m}$ telescope is $f / 6$. However, the fiber run from the $1 \mathrm{~m}$ telescope adds significant fiber to the standard $2.5 \mathrm{~m}$ configuration and includes several additional couplings, so the focal ratio degradation is expected to be a bit worse for the $1 \mathrm{~m}$ feed, which goes in the direction of making the beam more closely match that obtained with the $2.5 \mathrm{~m}$.

Figure 1 shows the profile of two bright sky lines, one in the long wavelength chip (chip 0) and one in the short wavelength chip (chip 2), for several $1 \mathrm{~m}$ and $2.5 \mathrm{~m}$ observations. This demonstrates that the line profiles as obtained with APOGEE from the two telescopes match extremely well. Comparison of spectra obtained for the same star from both telescopes also shows the same good match, although the different $\mathrm{S} / \mathrm{N}$ of the spectra make detailed comparison difficult. Both sky and stellar spectra suggest that the NMSU $1 \mathrm{~m}$ delivers extremely similar spectra to those delivered by the $2.5 \mathrm{~m}$ telescope.

\subsection{Target Selection}

Target selection is described in detail in Zasowski et al. (2013). The "main" sample is selected using a simple color cut with $(J-K)_{0}>0.5$, where colors are dereddened using a combination of near-IR and mid-IR photometry (Majewski et al. 2011), but there are also other target classes, including calibration and ancillary science program targets, as discussed in more detail in Zasowski et al. (2013).

For most of the stars, data are collected in multiple visits to allow detection of radial velocity variables. Standard survey targets are observed in multiple visits until a S/N of 100 per half-resolution element is achieved for a target of $H=12.2$. A minimum of three visits is required for most targets, with restrictions on the cadence so that a broad range of $\mathrm{RV}$ variables can be detected. However, survey software tracks accumulated $\mathrm{S} / \mathrm{N}$, and additional visits are added as needed to achieve the $\mathrm{S} / \mathrm{N}$ requirement. Some fainter targets (medium and long cohorts; see Zasowski et al. 2013) were observed for more than three visits. A subset of survey targets toward the Galactic Bulge was observed only in single visits (with a brighter limit for sample selection), because of the challenge associated with observing such targets from APO, where the Bulge can only be seen at high airmass.

\subsection{Scope of APOGEE Data Releases}

The APOGEE data in DR10 (Ahn et al. 2014) included the first year of APOGEE observations. The SDSS/APOGEE DR11 release added the second year of data, but was initially only released internally to SDSS collaboration members; it was publicly released along with DR12. Calibration of the DR10 and DR11 data is described in Mészáros et al. (2013).

The SDSS DR12 (Alam et al. 2015) includes all data taken with the APOGEE instrument between 2011 April and 2014 July. A total of 163,281 stars are included. Of these, 151,141 stars were observed during the main survey period that provided survey-quality spectra. Hot stars for telluric correction account for 15,343 of the survey-quality targets, and 17,116 of all targets (including commissioning data). Of the 135,798 survey-quality non-telluric sources, 110,087 come from the "main-survey" sample, which does not include special targets, targets from ancillary science programs, and stars observed with the NMSU $1 \mathrm{~m}$ (883 stars).

\section{BASIC DATA REDUCTION AND ASSOCIATED DATA PRODUCTS}

The basic data reduction procedure is described in Nidever et al. (2015). We provide a brief synopsis as an introduction to the data products that are included in the data releases and described here.

The raw data consist of data cubes for each individual exposure, where each layer of the cube is a read of the three detectors every $10.7 \mathrm{~s}$. As a result, the cubes are large, with a standard $500 \mathrm{~s}$ exposure consisting of 47 reads of 3 detectors, with each detector having $2560 \times 2048$ pixels, since a "reference" array (intended to help wth noise and crosstalk mitigation) of $512 \times 2048$ pixels is included. The raw data cubes are compressed using the standard FPACK routine (Seaman et al. 2010), but, to provide maximum compression, the raw data are converted to a series of differences between adjacent reads before compression. The resulting files are saved as $a p R$ files.

The reduction procedure first reduces these image cubes to individual 2D images. For each read, dark current is subtracted using a calibration product constructed from multiple dark images. Up-the-ramp sampling is then used to derive count rates that are less affected by readout noise than a simple doubly correlated sampling would provide. The resulting image 
Table 1

APOGEE Spectral Data Products

\begin{tabular}{ll}
\hline \hline Name & Contents \\
\hline apR-[abc]-ID8.apz & Raw data cubes $(2560 \times 2048 \times 47)$, APOGEE compressed format \\
ap2D-[abc]-ID8.fits & $2 \mathrm{D}(2048 \times 2048)$ dark subtracted, flat fielded \\
ap1D-[abc]-ID8.fits & 1D extracted $(2048 \times 300)$, rough wavelength calibration (not zero-pointed using sky) \\
apCframe-[abc]-ID8.fits & 1D spectra with sky subtraction, telluric correction, wavelength zeropoint adjustment \\
apVisit-APRED_VERS-PLATE-MJD-FIBER.fits & Individual visit spectra, dither-combined, but otherwise native pixel scale, \\
& one per object visit \\
apStar-APRED_VERS-APOGEE_ID.fits & Spectra resampled to constant $\Delta$ log $\lambda$, along with combined spectrum, one per object \\
aspcapStar-RESULTS_VERS-APOGEE_ID.fits & Normalized spectra to match those of spectral grids, \\
aspcapField-LOCATION_ID.fits & with best-matching grid spectra, one per object \\
& FITS tables with ASPCAP parameters and spectra \\
& for all stars in a given field \\
\hline
\end{tabular}

is flat fielded using a calibration product constructed from multiple flat-field images and is stored in an ap2D file.

Spectra are optimally extracted from the 2D images using an empirical PSF derived from an exposure of illuminated telescope mirror covers taken after the science exposure sequence has been completed. An approximate relative flux correction is also applied. These extracted, flux-calibrated spectra are saved as ap $1 D$ files, and the 2D models used for the extraction are saved as ap2Dmodel files.

The multiple exposures (ap $1 D$ files) are then combined to make an individual visit spectrum. To do this, the relative shifts between the different exposures, arising from the motion of the detector dither mechanism used to provide better sampling, are determined. Position-dependent sky subtraction is performed on individual exposures, as is a correction for telluric absorption. Exposures at different dither positions are paired, matching as best as possible exposures of comparable $\mathrm{S} / \mathrm{N}$, and combined into a spectrum with a sampling corresponding to half the original pixel sampling. The combination technique reduces to simple interleaving (after adjusting the levels of the two exposures to match) in the case of perfect 0.5 pixel dithers but properly handles dithers that are imperfect. The combined pairs are then coadded to provide the final visit spectra. An approximate absolute flux calibration is made to the spectrum using the 2MASS $H$ magnitude. An initial measurement of the radial velocity for each star in the visit is made by cross correlating each spectrum with the best match in a template library, and these are stored against the visit spectra in apVisit files.

All of the visits are then combined after resampling them to a common, log-lambda wavelength scale; in all data products, the wavelength scale is in vacuum wavelengths. Relative radial velocities of each visit are iteratively refined by crosscorrelation of each visit spectrum with the combined spectrum (which should provide a perfect template match). The absolute radial velocity scale is then set by cross-correlating the combined spectrum with the best match in a template library. The resulting combined spectrum, as well as the resampled visit spectra, are stored in apStar files.

A summary of the spectral data products is presented in Table 1. The DR12 Science Archive Server (http://data.sdss3. org/sas/dr12) makes both raw data, as well as various stages of reduced data, available to the community, as discussed in Section 6. More details on the files are presented in Section 8.

The reduction pipeline software is version-controlled in the SDSS-III software repository.

\section{SPECTROSCOPIC ANALYSIS OF APOGEE DATA AND ASSOCIATED DATA CATALOGS}

Stellar parameters and individual chemical abundances are derived from the combined APOGEE spectra with the APOGEE Stellar Parameters and Chemical Abundances Pipeline (ASPCAP), which is described in detail in A. E. García Pérez et al. (2015, in preparation). The basic idea is that a grid of synthetic spectra is searched (with interpolation in the grid) to find the best match to each observed spectrum, and the parameters of the best match are adopted as the best parameters for that star.

\subsection{Synthetic Spectra}

Central to ASPCAP is the construction of a synthetic spectral grid that covers the range of fundamental stellar parameters over which main survey stars are expected to be found. Given the significance and frequency of molecular features-in particular, from $\mathrm{CO}, \mathrm{CN}$, and $\mathrm{OH}-$ in the region of the $\mathrm{H}$ band where APOGEE spectra are recorded, the dimensions of this grid include not only the usual effective temperature $\left(T_{\text {eff }}\right)$, surface gravity $(\log g)$, microturbulent velocity $\left(v_{\text {micro }}\right)$, and overall scaled-solar chemical abundance $([\mathrm{M} / \mathrm{H}])$, but also the carbon $([\mathrm{C} / \mathrm{M}])$, nitrogen $([\mathrm{N} / \mathrm{M}])$, and $\alpha$-element abundances $([\alpha / \mathrm{M}]$, which includes oxygen) relative to the solar abundance ratio. The resulting $7 \mathrm{D}$ grid comprises several million individual synthetic spectra.

The fidelity of the synthetic spectral library is critical to the success of the method. The structures of the underlying stellar atmospheres were computed with the ATLAS9 code (Kurucz 1979). For DR10/DR11, a grid of atmospheres was used (Castelli \& Kurucz 2004) that adopted solar-scaled abundances (i.e., the carbon, nitrogren, and $\alpha$ abundances were varied only in the spectral synthesis, but not in the underlying atmospheric model structure). For DR12, a self-consistent set of atmospheres was used: carbon and $\alpha$ abundances were varied across the grid for the atmosphere calculation (the nitrogen abundance has little effect on the structure of the atmosphere, so this was not varied). Details are given in Mészáros et al. (2012). These calculations assume plane-parallel atmospheres (but see Zamora et al. 2015 for some discussion of the changes resulting from using a spherical geometry) in local thermodynamic equilibrium (LTE).

The synthetic spectra were generated using the LTE-1D branch of the ASS $\epsilon$ T synthesis code (Koesterke 2009). A custom line list was compiled as discussed in M. Shetrone et al. (2015, in preparation). The atomic and molecular data for the 
line list were taken from various literature sources, but the atomic data transition probabilities were adjusted, within limits, by matching synthetic spectra for the Sun and Arcturus with available observed high-resolution atlases (Livingston \& Wallace 1991; Hinkle et al. 1995) as described in M. Shetrone et al. (2015, in preparation). The solar abundances assumed for these adjustments are those from Asplund et al. (2009) and Asplund et al. (2005) (for DR10/11 and DR12, respectively) and those for Arcturus from consideration of a number of sources; the adopted values for the various APOGEE line lists are presented in M. Shetrone et al. (2015, in preparation). As described in M. Shetrone et al. (2015, in preparation), the linelist has evolved, and future development is likely. Additional details on the spectral grids and a comparison of the ATLAS9/ASS $\epsilon$ T synthetic spectra with those from other atmospheres and syntheses are discussed in Zamora et al. (2015).

The high-resolution synthetic spectra are then matched to the APOGEE resolution and pixel sampling. This is accomplished by smoothing the high-resolution spectrum with the line-spread function (LSF) of APOGEE data. However, the APOGEE LSF varies across the detector as a consequence of the optical design. To some extent, this variation is reduced when multiple visits are combined, since stars will generally land in a different location on the detector (from a different fiber in the telescope focal plane) in different visits. In DR10, a Gaussian LSF with $R=22,500$ was adopted, but we subsequently recognized that the APOGEE LSF is not well-represented by a Gaussian. For DR12 we have taken the approach of using an LSF made by averaging five different empirically measured LSFs across the detector to make a single library for use with all of the SDSSIII/APOGEE spectra. As demonstrated below, this yields results that are generally validated by observations of objects of known abundances, but it is an area for potential improvement in subsequent data releases. A. E. García Pérez et al. (2015, in preparation) present a more detailed investigation of the potential impact of assuming an imperfect LSF.

In addition to smoothing the high-resolution synthetic spectra with an APOGEE LSF, in DR12 we also include broadening to account for macroturbulent velocity in the stellar atmosphere. We were motivated to do this not only because it is a known physical phenomenon, but also because this additional broadening brings our derived surface gravities closer to those suggested by other techniques (see below). For DR12, we have adopted a simple scheme of broadening with a Gaussian of FWHM $6 \mathrm{~km} \mathrm{~s}^{-1}$ for the macroturbulent velocity, motivated by typical values from Carney et al. (2008). Adopting a different functional form, along with possible variations in macroturbulent velocity as a function of other stellar parameters, is another area for potential improvement in the future.

The synthetic spectra are calculated relative to a true continuum. However, it is challenging, if not impossible, to identify a true continuum in the observed spectra in cool, metal-rich stars. To account for this, both the synthetic spectra and the observed spectra are "pseudo-continuum" normalized using the same algorithm, as discussed in A. E. García Pérez et al. (2015, in preparation).

The smoothed, resampled, and pseudo-continuum normalized spectra are bundled into a large library. To minimize the (substantial) computing resources required for such a large grid, we break it into two sub-grids overlapping in temperature (which we denote GK and F); we find the best match in each grid, and use the result that provides the better match. In addition, the libraries are compressed using a principal components decomposition, in which we split the spectra into 30 pieces and PCA-compress each of those, keeping only the first 30 principal components and the amplitudes of these for each location in the grid; additional details are provided in A. E. García Pérez et al. (2015, in preparation).

The range and spacing of the ATLAS9/ASS $\epsilon$ T grid parameters used for DR12 are given in Table 2 (see also Zamora et al. 2015). A. E. García Pérez et al. (2015, in preparation) discuss why this grid spacing is adequate and how interpolation within the grid is performed.

We note that for SDSS-III/APOGEE, stellar rotation is not included in the synthetic spectral grids. For stars with rotational velocities $\left(\geqslant 5 \mathrm{~km} \mathrm{~s}^{-1}\right)$ that broaden the lines significantly beyond the instrument resolution, the libraries will not be able to obtain a good match to the observed spectra, and the resulting stellar parameters can be affected systematically. Stellar rotation is not expected to be significant for most of the red giants that make up the APOGEE sample. However, a significant fraction of the dwarfs observed in the survey may have detectable rotation, and results for these that do not model rotation are suspect. As discussed below, we do not provide calibrated parameters for dwarfs, partly because of the lack of treatment of rotation. For all survey stars, we provide a flag that indicates whether rotation is likely to be important, based on a comparison of the width of the autocorrelation function of the spectrum relative to the autocorrelation of the best-matching RV template. Future improvements are planned with the inclusion of rotation when needed.

We note that the current analysis is limited to stars with $T_{\text {eff }}$ $>3500 \mathrm{~K}$ because the Kurucz grid of model atmospheres does not extend to cooler temperatures. Subsequent work is planned to extend results to cooler temperatures using MARCS model atmospheres and the Turbospectrum synthesis code (Zamora et al. 2015).

The current grid has a range in metallicity from $-2.5<[\mathrm{M} /$ $\mathrm{H}]<0.5$, so this imposes the range over which ASPCAP can provide metallicities. Stars with derived parameters at the grid edges may have true parameters that lie outside the grid, and they are flagged as having unreliable parameters.

\subsection{Deriving Stellar Parameters and Abundances}

For each APOGEE spectrum, a best match within the synthetic grid is found. To do this, interpolation within the grid, as well as an efficient search method, are required. This is accomplished using the FORTRAN95 code FERRE (Allende Prieto et al. 2006), ${ }^{31}$ which yields a best set of stellar parameters. The computational resources to perform the full 7D search for $>100,000$ stars are substantial $(>50,000 \mathrm{CPU}$ hours). Because of this, we use a calibration subsample (see Section 5) to derive a relation for the microturbulent velocity as a function of surface gravity. Figure 2 shows the derived microtubulent velocity as a function of surface gravity for the calibration subsample, color-coded by metallicity. The stars of higher metallicity that make up the bulk of the APOGEE sample (but not the calibration sample) define a fairly tight sequence. We a fit a linear relation to the subset of stars from

\footnotetext{
31 http://www.sdss3.org/svn/repo/apogee/aspcap/ferre, http://hebe.as. utexas.edu/ferre
} 
Table 2

Synthetic Spectral Grid Parameter Values

\begin{tabular}{lccc}
\hline \hline Gridname & Dimension & Range & Step Size \\
\hline GK grid & $T_{\text {eff }}$ & 3500 to 6000 & 250 \\
& $\log g$ & 0 to 5 & 0.5 \\
& $\log v_{\text {micro }}$ & -0.301 to 0.903 & 0.301 \\
& {$[\mathrm{M} / \mathrm{H}]$} & -2.5 to 0.5 & 0.5 \\
& {$[\mathrm{C} / \mathrm{M}]$} & -1 to 1 & 0.25 \\
& {$[\mathrm{~N} / \mathrm{M}]$} & -1 to 1 & 0.5 \\
& {$[\alpha / \mathrm{M}]$} & -1 to 1 & 0.25 \\
F grid & $T_{\text {eff }}$ & 5500 to 8000 & 250 \\
& $\log g$ & 1 to 5 & 0.5 \\
& $\log v_{\text {micro }}$ & -0.301 to 0.903 & 0.301 \\
& {$[\mathrm{M} / \mathrm{H}]$} & -2.5 to 0.5 & 0.5 \\
& {$[\mathrm{C} / \mathrm{M}]$} & -1 to 1 & 0.25 \\
& {$[\mathrm{~N} / \mathrm{M}]$} & -1 to 1 & 0.5 \\
& {$[\alpha / \mathrm{M}]$} & -1 to 1 & 0.25 \\
& &
\end{tabular}

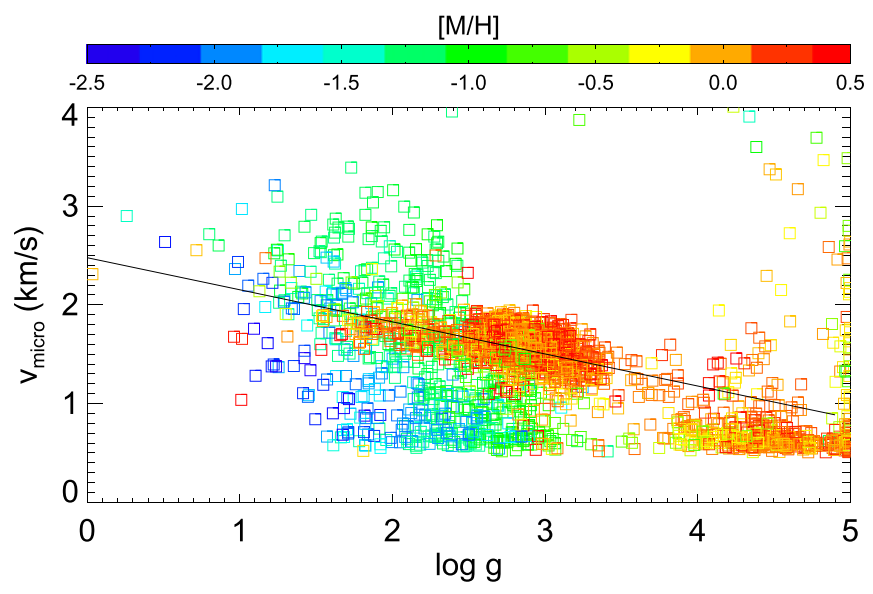

Figure 2. Microturbulence relation, as derived from a 7D run of the calibration subsample.

these data with $\log g<3.8$ and $[\mathrm{M} / \mathrm{H}]>-1$ and derived the fit that was adopted for DR12:

$$
v_{\text {micro }}=2.478-0.325 \log g \text {, }
$$

which is shown as a solid line in Figure 2. The rms scatter around this relation is $0.22 \mathrm{~km} \mathrm{~s}^{-1}$ for stars with $\log g<3.8$, and $0.83 \mathrm{~km} \mathrm{~s}^{-1}$ if all stars are included.

We use this relation to construct a $6 \mathrm{D}$ grid, interpolating within the 7D grid to the microturbulent velocity appropriate for the surface gravity of each grid point. The utility of this is that it reduces the search to a $6 \mathrm{D}$ one, rather than $7 \mathrm{D}$, with a corresponding reduction in the time required (by a factor of three or more) to analyze the full sample.

The full DR12 sample is then analyzed using the $6 \mathrm{D}$ grid to provide the initial set of parameters: $T_{\text {eff }}, \log g,[\mathrm{M} / \mathrm{H}],[\mathrm{C} / \mathrm{M}]$, $[\mathrm{N} / \mathrm{M}]$, and $[\alpha / \mathrm{M}]$.

Given the stellar parameters, the spectra are then analyzed for abundances of each of 15 different elements $-\mathrm{C}, \mathrm{N}, \mathrm{O}, \mathrm{Na}$, $\mathrm{Mg}, \mathrm{Al}, \mathrm{Si}, \mathrm{S}, \mathrm{K}, \mathrm{Ca}, \mathrm{Ti}, \mathrm{V}, \mathrm{Mn}, \mathrm{Fe}$, and $\mathrm{Ni}$ - that have features in the APOGEE wavelength coverage. For each element, the regions in the spectrum that are sensitive to the abundance of that element, and less sensitive to the abundance of other elements, are determined from model spectra. The spectral "windows" identified in this way are weighted by their relative sensitivities and also by how well a synthetic spectrum with the parameters appropriate to Arcturus match those of an observed Arcturus spectrum. Details of this procedure, and the specific locations of the derived windows, are discussed in A. E. García Pérez et al. (2015, in preparation). The amount of spectrum covered by the windows varies dramatically across the elements, from elements where there are only a handful of lines (e.g., $\mathrm{Na}, \mathrm{S}, \mathrm{K}, \mathrm{V}$ ) to elements that affect a significant fraction of the entire APOGEE spectra $(\mathrm{C}, \mathrm{N}, \mathrm{O}, \mathrm{Si}, \mathrm{Fe})$.

Given the windows for each element, each spectrum is then run through the FERRE search code separately for each element, using the same grid as used for the determination of the stellar parameters. However, for the individual element abundances, the search is done holding the stellar parameters $T_{\text {eff }}, \log g, v_{\text {micro }}$ fixed, and just varying a single metallicity parameter, so this is a one-dimensional search. For $\mathrm{C}$ and $\mathrm{N}$, the $[\mathrm{C} / \mathrm{M}]$ and $[\mathrm{N} / \mathrm{M}]$ dimension is varied, for the $\alpha$ elements $(\mathrm{O}$, $\mathrm{Mg}, \mathrm{Si}, \mathrm{S}, \mathrm{Ca}$, and $\mathrm{Ti})$, the $[\alpha / \mathrm{M}]$ dimension is varied, while for all others ( $\mathrm{Na}, \mathrm{Al}, \mathrm{K}, \mathrm{V}, \mathrm{Mn}, \mathrm{Fe}, \mathrm{Ni})$, the $[\mathrm{M} / \mathrm{H}]$ dimension is varied. Apart from $\mathrm{C}$ and $\mathrm{N}$, this means that more than just the single element abundance being sought is being varied, but ASPCAP attempts to account for that by using windows with maximal sensitivity to the element being derived and minimal sensitivity to other abundances. This allows us to avoid having to create a series of 15 different $7 \mathrm{D}$ grids where pure individual element variations are calculated.

\subsection{FERRE Output}

The full ASPCAP pipeline produces an initial set of six parameters for each star from the initial $6 \mathrm{D}$ fit, plus 15 additional abundance measurements. The raw output from the 6D fit are stored in an array called FPARAM, while the output from the 15 separate 1D "window" fits are stored in an array called FELEM.

FERRE also produces a covariance matrix from the initial 6D fit, which we store in a matrix called FPARAM_COVAR, and individual abundance uncertainty estimates, which we store in a FELEM_ERR array. Unfortunately, we have found that the formal errors returned by FERRE seem to be significantly underestimated when they are calculated using standard fit techniques (i.e., inverse of the curvature matrix); this may arise because systematic errors (e.g., in the synthetic calculations, LSF-matching, etc.) dominate statistical ones (see A. E. García Pérez et al. 2015, in preparation for more details). As a result, we also determine and store a more empirical estimate of uncertainties, as discussed below.

For each parameter and elemental abundance, we store a bitmask flagging possible conditions encountered in the process, such as a solution falling near the edge of the grid, and this mask can be used to identify abundances that may be unreliable.

The data products are discussed in more detail in Sections 68. The ASPCAP software is version controlled in the SDSS-III software repository in the speclib, idlwrap and ferre products.

\subsection{Versions and Data Releases}

There are four main stages of APOGEE data processing: visit reduction, star combination, ASPCAP/FERRE processing, and final calibration. The software is set up such that these four stages can be run with independent software versions/ configurations so that, for example, a later stage can be run 
Table 3

Software Version and Product Names

\begin{tabular}{lcccc}
\hline \hline Data Release & APRED_VERS (apogeereduce) & APSTAR_VERS (apogeereduce) & ASPCAP_VERS (idlwrap) & RESULTS_VERS (idlwrap) \\
\hline DR10 & r3 (v2_11) & s3 (v2_11) & a3 (v7) & v304 (v15) \\
DR11 & r4 (v2_14) & s4 (v2_15) & a4 (v16) & v402 (-) \\
DR12 & r5 (v3_04) & stars (v3_10) & 125_6d (v22) & v603 (v27) \\
\hline
\end{tabular}

without having to rerun an earlier stage with a new software version. The output from the four stages is saved in a series of nested subdirectories, as described in Section 6. The names of the different versions are tied to software version numbers of the different products. Table 3 gives the names and versions used for the APOGEE data releases.

\section{CALIBRATION AND VALIDATION OF DR12 PARAMETERS AND ABUNDANCES}

Results for the stellar parameters and abundances have been calibrated and validated based on observations of a calibration sample. This sample includes stars in the Kepler field that have well-characterized effective temperatures and surface gravities, samples of stars in a set of globular and open clusters that span a wide range of metallicity, and a set of stars with previously measured parameters and abundances.

\subsection{Effective Temperatures}

We compare the ASPCAP spectroscopically determined temperatures with those obtained from the calibrated photometric relation of González Hernández \& Bonifacio (2009). We chose a sample of giants $(\log g<3.8)$ in the Kepler field and adopted $E(B-V)$ estimates as determined from the SAGA survey (Casagrande et al. 2014). We convert $E(B-V)$ to $E(J-K)$ using $E(J-K)=\left(0.56+0.06(J-K)_{0}\right) * E(B-V)$ (Bessell et al. 1998). We use the dereddened $J-K$ color to derive the temperature, and adopt the raw ASPCAP metallicity for the color-temperature relation. We restrict the comparison to stars with ASPCAP metallicity $[\mathrm{M} / \mathrm{H}]>-1$. Figure 3 shows the difference between the spectroscopic and photometric temperatures for this subsample. This comparison suggests that there is an offset in the derived effective temperature in that the raw ASPCAP temperature is about $90 \mathrm{~K}$ cooler than the photometric temperatures.

We derived a calibration based on a linear fit with temperature to these data, which yields:

$$
T_{\text {eff,corr }}=T_{\text {eff,raw }}-0.0034\left(T_{\text {eff,raw }}-4500\right)+87.9
$$

although the dependence of the difference on temperature is negligibly small.

The calibrated temperature provided for all giants in the DR12 release uses the calibration from Equation (2). For giants with effective temperatures outside the range of those used to derive the calibration $\left(3500<T_{\text {eff }}<6000\right)$, the correction at the edge of the range was adopted, and the CALRANGE_WARN bit has been set in the PARAMFLAG for temperature (see Section 7).

An upper limit on the temperature uncertainty is derived from the scatter around this relation, which gives $\sigma\left(T_{\text {eff }}\right)=91.5 \mathrm{~K}$. This is an upper limit because there are uncertainties associated with the photometry; an uncertainty of 0.02 mag in $(J-K)_{0}$ typically gives an uncertainty of $\sim 50 \mathrm{~K}$. Nonetheless, the $91.5 \mathrm{~K}$ scatter around the calibration relation

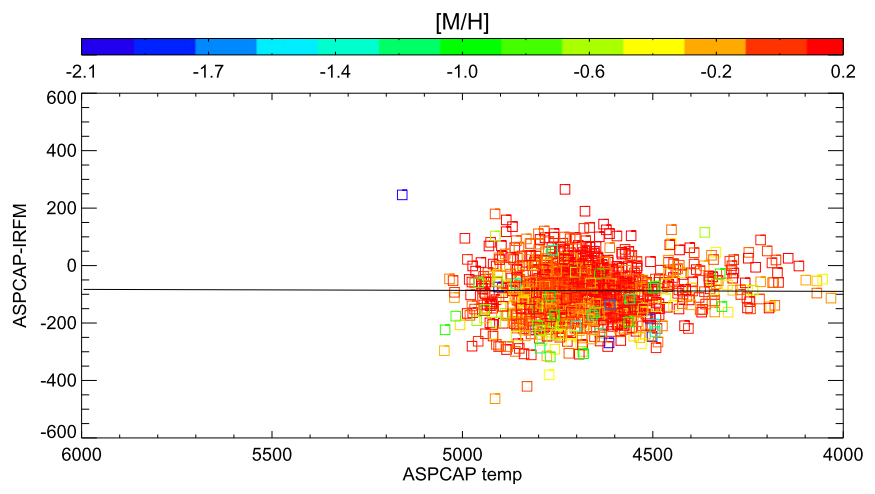

Figure 3. Temperature calibration, derived from low-reddening sample with photometric temperatures. The scatter around this relation, $\sigma\left(T_{\text {eff }}\right)=91.5 \mathrm{~K}$, gives an upper limit to the temperature uncertainty.

was adopted as the empirical temperature uncertainty for stars in DR12. There may be also be some systematic uncertainties related with the photometric temperature relation; as discussed by González Hernández \& Bonifacio (2009), differences in temperature scales between different studies can be as large as $100 \mathrm{~K}$.

The color-temperature relation of González Hernández \& Bonifacio (2009) is also used to flag objects that may have poor stellar parameters. For each object, a temperature is determined using the color-temperature relation, the 2MASS $J-K$ color, and the estimate of the reddening used by the targeting pipeline. This is compared with the ASPCAP-derived temperature. When these deviate by more than $1000 \mathrm{~K}$, the COLORTE_WARN bit is set in the ASPCAPFLAG (see Section 7); if the temperature differ by more than $2000 \mathrm{~K}$, the COLORTE_BAD bit is set and the ASPCAP results are flagged as bad. Most of these are cool dwarfs that are not fit well (in retrospect, we might have chosen less conservative values to flag).

\subsection{Surface Gravities}

Surface gravity is a key parameter for a number of reasons: (1) it is important for estimating distances to survey stars, (2) it can be used to identify AGB stars, and (3) it can have a significant effect on line strengths.

The APOGEE survey includes observations of giants that have time series photometry with Kepler that allows asteroseismic analysis. In principle, such analysis very accurate surface gravities, with an estimated uncertainty of $\ll 0.05$ in $\log g$ (Hekker et al. 2013).

A calibration relation for surface gravity has been derived using a subset of stars from version 7.3 of the APOKASC catalog (Pinsonneault et al. 2014). To cover a wide range of properties, we took all stars that had inferrable evolutionary states from Stello et al. (2013), all stars with $\log g<2$, and all of the small number of stars in the APOKASC catalog with $[\mathrm{M} / \mathrm{H}]<-1$. 


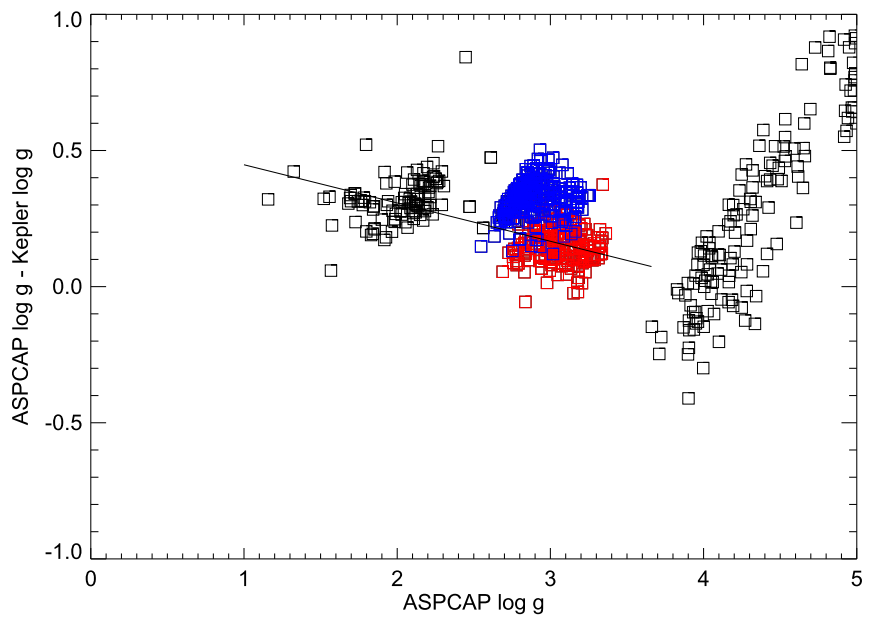

Figure 4. Surface gravity calibration, derived from a sample of stars in the Kepler field with gravities from asteroseismic analysis. This analysis also provides information on the evolutionary state: red points are hydrogen shell burning (RGB) stars, while blue points are helium core burning (RC) stars.

Figure 4 shows the difference between ASPCAP spectroscopy $\log g$ and the asteroseismic $\log g$ from Pinsonneault et al. (2014), as a function of surface gravity. It is clear that results for stars at higher surface gravity $(\log g \geqslant 4)$ are poor. We believe that this is caused to some extent by the lack of treatment of rotation discussed above.

At lower surface gravities, the spectroscopic surface gravities are systematically higher than the asteroseismic surface gravities. In addition, there are systematic offsets between gravities determined for RGB and RC stars; the difference between ASPCAP and asteroseismic gravities is larger for RC stars, even for $\mathrm{RGB} / \mathrm{RC}$ pairs at the same gravity and metallicity. This has been noted in previous APOGEE samples (Mészáros et al. 2013; Pinsonneault et al. 2014), but the effect is still currently not well understood. If one considers just the RGB sample, it appears that the difference between ASPCAP and asteroseismic gravity increases to lower gravities. No dependence on metallicity was found.

We have chosen to adopt a surface gravity calibration using a linear surface gravity fit to the RGB sample. If one assumes that the asteroseismic gravities are correct, this would imply that the RC stars in the DR12 sample will have calibrated surface gravities that are too large by $\sim 0.2$ in $\log g$. We have chosen this approach rather than "splitting the difference" between the RGB and RC samples because it is possible to distinguish the bulk of the RC stars from RGB stars based on cuts in temperature and surface gravity (see Bovy et al. 2014). Because RC stars are expected to have well-constrained luminosities, it is possible to use these for distance estimates (Bovy et al. 2014) instead of needing to rely on the ASPCAP surface gravities. We thus choose to adopt the calibration relation that we hope will give the best results for RGB stars, and recommend treating the RC stars separately. The derived calibration relation is given by:

$$
\log g_{\text {corr }}=\log g_{\text {raw }}+0.14 \log g_{\text {raw }}-0.588 .
$$

The scatter around this relation is $\sim 0.08$ in $\log g$. For empirical uncertainties in the $\log g$ released in DR12, we adopt the scatter around the mean difference between the spectroscopic $\log g$ and the asteroseismic $\log g$ (again, ignoring the $\mathrm{RC}$ stars), which gives an uncertainty of 0.11 in $\log g$.
However, the true error is probably more likely to be dominated by systematics, given the Kepler differences. We have also determined that the surface gravity is sensitive to the details of the LSF used in the analysis, so this may lead to systematics depending on the exact LSF combination of each spectrum.

We hope that future work will lead to a better understanding of potential issues with the spectroscopic surface gravities.

\subsection{Calibrated Parameters and Abundance Determination}

Our procedure to determine elemental abundances uses the raw derived stellar parameters when fitting synthetic spectra to the windows in the spectra that cover features of specific elements. We choose to do this because (1) using calibrated parameters would lead to significantly poorer overall spectrum fits, and (2) it is possible that the raw, "spectroscopic," parameters account to some extent for uncertainties and approximations that are implicit in the synthetic spectra, so using the raw parameters provides a more self-consistent set of abundances.

On the other hand, it is also possible that the raw parameters are offset for other reasons, for example, if the adopted LSF leads to incorrect surface gravities. If this were the case, then one might argue that using the calibrated parameters for individual elemental abundances might be more accurate, at least for features where the strength depends strongly on surface gravity. Future work is planned to investigate this.

\subsection{Chemical Abundances}

Our primary calibration sample for abundances is a set of globular and open clusters spanning a wide range of metallicity, from M92 and M15 on the metal-poor end ([Fe/ $\mathrm{H}]=-2.35$ and -2.33$)$ to NGC 6791 on the metal-rich end $([\mathrm{Fe} / \mathrm{H}]=0.37)$. The set of calibration clusters is presented in Table 4, where we also note the number of likely members used for the APOGEE calibration.

We have approached calibration in two steps: an internal calibration where we look for homogeneity of elemental abundances within clusters, and an external calibration where we investigate the agreement of the abundances with previously measured values.

\subsubsection{Internal Calibration}

For the internal calibration, we examine the ASPCAP abundances under the assumption that clusters are chemically homogeneous for all elements except carbon and nitrogen, which are expected to have variations in giants because of potential mixing of CNO-processed material. The sample was restricted to more metal-rich clusters because (1) multiple populations in some globular clusters are manifest in elemental abundances (e.g., $\mathrm{Al}, \mathrm{Mg}, \mathrm{Na}$, and $\mathrm{O}$ ), and our cluster sample is dominated by globular clusters at $[\mathrm{M} / \mathrm{H}]<-1$; (2) at lower metallicity, the lines are weaker, so trends are more challenging to discern; and (3) the bulk of the main APOGEE sample is at higher metallicity, so we choose to provide an internal calibration that is most accurate for the bulk of the sample. In practice, we inspected the spread of each individual element within clusters of various metallicities, and chose a lower metallicity limit for each element to use for the internal calibration; this limit was always between $[\mathrm{M} / \mathrm{H}]$ of -1 and -0.6 . 
Table 4

Calibration Clusters

\begin{tabular}{lccc}
\hline \hline Cluster & $N$ & Literature $[\mathrm{Fe} / \mathrm{H}]$ & Reference \\
\hline M92 & 43 & $-2.35 \pm 0.05$ & Carretta et al. (2009) \\
M15 & 40 & $-2.33 \pm 0.02$ & Carretta et al. (2009) \\
M53 & 13 & $-2.06 \pm 0.09$ & Carretta et al. (2009) \\
N5466 & 9 & $-2.01 \pm 0.09$ & Lamb et al. (2015) $^{\mathrm{a}, \mathrm{b}}$ \\
N4147 & $0^{\mathrm{c}}$ & $-1.78 \pm 0.08$ & Carretta et al. (2009) \\
M2 & 10 & $-1.66 \pm 0.08$ & Carretta et al. (2009) \\
M13 & 85 & $-1.58 \pm 0.04$ & Carretta et al. (2009) \\
M3 & 70 & $-1.50 \pm 0.05$ & Carretta et al. (2009) \\
M5 & 91 & $-1.33 \pm 0.02$ & Carretta et al. (2009) \\
M107 & 39 & $-1.03 \pm 0.02$ & Carretta et al. (2009) \\
M71 & 26 & $-0.82 \pm 0.02$ & Carretta et al. (2009) \\
Be29 & 6 & $-0.44 \pm 0.18$ & Carraro et al. (2004) \\
N2243 & $0^{\mathrm{c}}$ & $-0.35 \pm 0.05$ & Jacobson et al. (2011a) $^{\mathrm{d}}$ \\
N2158 & 17 & $-0.21 \pm 0.05$ & Jacobson et al. (2011b) $^{\mathrm{d}}$ \\
M35 & $0^{\mathrm{d}}$ & $-0.14 \pm 0.10$ & Barrado Navascués et al. (2001) \\
N2420 & 15 & $-0.13 \pm 0.06$ & Jacobson et al. (2011b) $^{\mathrm{d}}$ \\
Pleiades & $0^{\mathrm{a}}$ & $0.03 \pm 0.02$ & Soderblom et al. (2009) \\
N188 & 6 & $0.04 \pm 0.04$ & Jacobson et al. (2011b) $^{\mathrm{d}}$ \\
M67 & 40 & $0.06 \pm 0.05$ & Jacobson et al. (2011b) $^{\mathrm{d}}$ \\
N7789 & 5 & $0.09 \pm 0.04$ & Jacobson et al. (2011b) \\
N6819 & 37 & $0.16 \pm 0.03$ & Bragaglia et al. (2001) \\
N6791 & 28 & $0.37 \pm 0.05$ & Carraro et al. (2006) \\
\hline & & &
\end{tabular}

Notes.

a Observed stars are dwarfs, which have not bee used for calibration in DR12.

${ }^{\mathrm{b}}$ Lamb et al. (2015) give -2.04; our value was from a in-preparation draft.

${ }^{\mathrm{c}}$ Cluster observed, but not used for DR12 calibration.

$\mathrm{d}$ Adjusted by +0.07 dex to compensate for literature adoption of solar $\mathrm{Fe}$ abundance of 7.52 , while we adopt 7.45 .

For the internal calibration, we look for agreement among the cluster stars, without any constraints on what the mean abundances are. Inspection of the raw ASPCAP abundances within each cluster suggests that the abundances of some elements appear to have small trends with effective temperature. To quantify this effect we fit, for each element, for mean abundances of each cluster plus a linear temperature dependence of the abundance:

$$
\left[\mathrm{X}_{i j} / \mathrm{H}\right]=\left[\mathrm{X}_{i} / \mathrm{H}\right]+S_{\mathrm{X}}\left(T_{i j}-4500\right) / 1000
$$

where $i$ represents the cluster, and $j$ the star within the cluster, and $S_{\mathrm{X}}$ the derived slope for element X. Note that we used [X/ $\mathrm{H}]$ for all abundances: for the elements for which ASPCAP uses a grid dimension that is relative to the overall metal abundance (C, N, and $\alpha$ elements), we added the ASPCAP [M/ $\mathrm{H}]$ to get $[\mathrm{X} / \mathrm{H}]$. This made it easier to detect potential cluster non-members as those stars with abundances significantly different from the cluster averages. We attempted to be conservative about cluster membership: we started with stars selected to be probable cluster members (see Zasowski et al. 2013), but then adopted only stars with radial velocities within $5 \mathrm{~km} \mathrm{~s}^{-1}$ of our cluster mean, and then finally rejected stars with abundance residuals more than 0.2 dex from the fit.

Results are shown in Figure 5, where abundances of each cluster calibration star are shown relative to the derived mean $\left(\left[\mathrm{X}_{i} / \mathrm{H}\right]\right)$ of the cluster of which it is a member. Different colors and point styles identify the clusters to which stars belong. The small temperature trends could arise from a number of different factors: the assumption of LTE, issues with atomic data since different lines provide information about abundances at different temperatures, etc. The data suggest that a linear temperature term is adequate to describe the trends for most elements.

For DR12, we have adopted the derived temperature trends for an internal calibration of the abundances for all elements except carbon and nitrogen. The slopes of these temperature terms $\left(S_{\mathrm{X}}\right)$ are given in Table 5 . We arbitrarily define the abundance of a given element as the abundance at $T_{\text {eff }}$ $=4500 \mathrm{~K}$, which could lead to some small zeropoint offsets in the abundances. While is it possible that the derived abundances of carbon and nitrogen may have temperature dependencies, we cannot discern these from the cluster data internally.

We apply the temperature corrections as derived from the cluster sample to the DR12 sample. Because the clusters only provide stars with $3800<T_{\text {eff }}<5250 \mathrm{~K}$, we do not extrapolate the linear relation outside this range. Instead, we use the correction at $T_{\text {eff }}=5250 \mathrm{~K}$ for all stars with $T_{\text {eff }}>5250 \mathrm{~K}$, and the correction at $T_{\text {eff }}=3800 \mathrm{~K}$ for all stars with $T_{\text {eff }}<3800 \mathrm{~K}$. For those giants outside the temperature range of the calibrator, we set the CALRANGE_WARN bit in the abundance masks.

In addition, because the calibration is derived only from giants, and because of other issues noted above for dwarfs, we do not apply it to stars with $\log g>3.8$. For these higher surface gravity stars, we do not provide calibrated abundances at all (the values are set to -9999) and we set the CALRANGE_BAD bit in the abundance masks. The raw ASPCAP parameters are still provided.

\subsubsection{Empirical Abundance Precision}

The individual abundances of stars in clusters provide an excellent data set to assess the precision of our derived abundances, by using the scatter within clusters as an estimate of the abundance uncertainties. This scatter is a function of both temperature and metallicity, which is as expected because lines are weaker at lower metallicity, and change strength with temperature. In addition, we see that scatter decreases with increasing signal-to-noise.

To derive empirical uncertainties, we calculated the spread of the abundance within each cluster in bins of width $250 \mathrm{~K}$ in temperature and 50 in $\mathrm{S} / \mathrm{N}$ (putting all observations with $\mathrm{S} / \mathrm{N}$ $>200$ into the highest $\mathrm{S} / \mathrm{N}$ bin). To achieve a greater range in $\mathrm{S} / \mathrm{N}$, we included abundance measurements for cluster stars derived from individual visits in addition to those from the combined spectra. We combined results for all clusters in bins of 0.5 dex in metallicity, and then fit a simple functional form to the uncertainty in each element:

$$
\begin{aligned}
\ln \left(\sigma_{\mathrm{X}}\right)= & A_{\mathrm{X}}+B_{\mathrm{X}}\left(T_{\mathrm{eff}}-4500\right) / 1000 \\
& +C_{\mathrm{X}}[\mathrm{M} / \mathrm{H}]+D_{\mathrm{X}}(\mathrm{S} / \mathrm{N}-100)
\end{aligned}
$$

where we fit for the natural log to ensure that the uncertainty never goes negative (i.e., outside of the range of the calibration cluster data).

Table 6 gives the coefficients for these empirical error estimates for all of the abundances. The second-to-last column gives the empirical error estimate at $T_{\text {eff }}=4500 \mathrm{~K}$, solar metallicity, and $\mathrm{S} / \mathrm{N}=100$. For all elements except vanadium, 


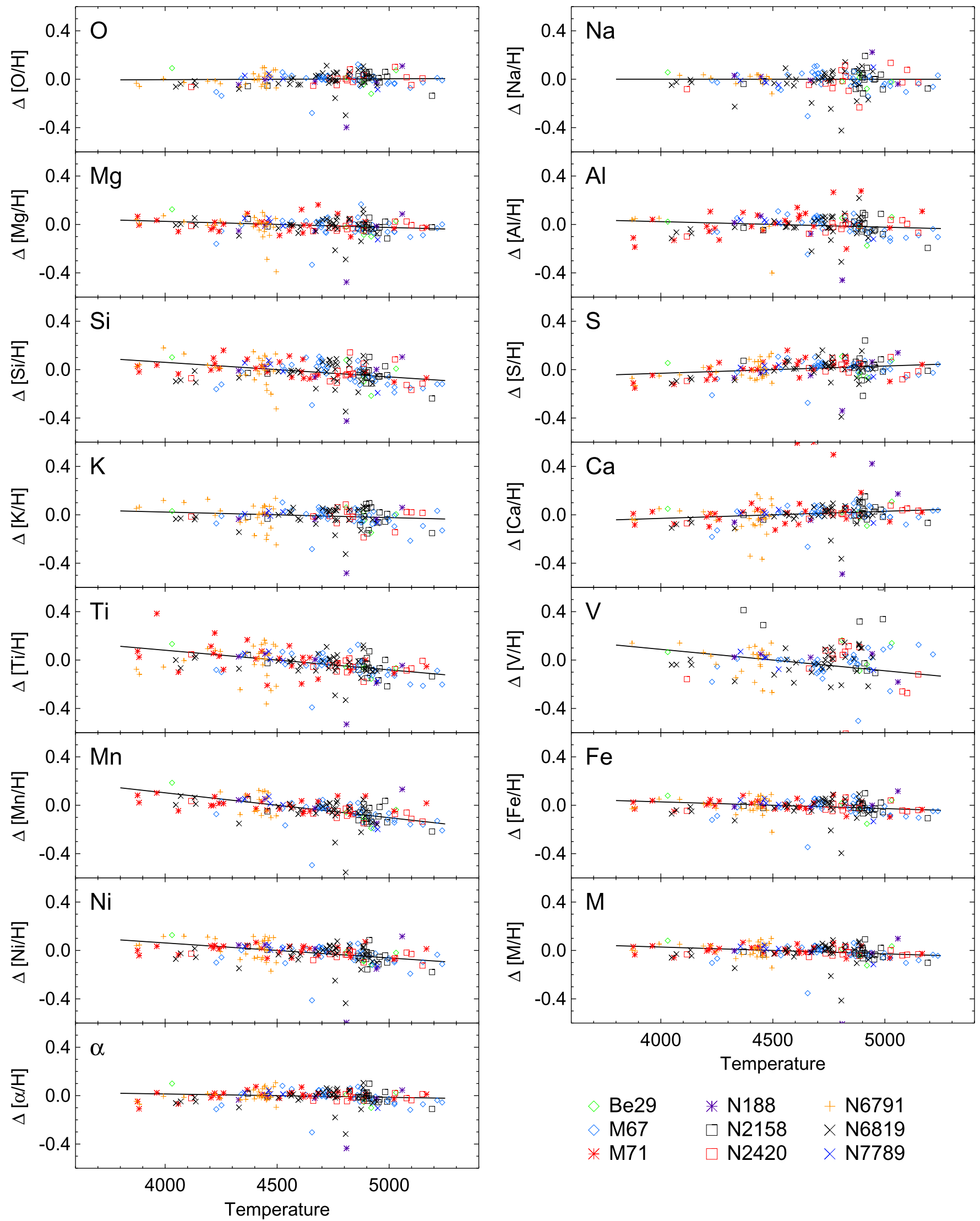

Figure 5. Internal elemental abundance calibrations. Plots show deviations of individual elements relative to the cluster means as a function of temperature; these have been defined to be zero at $4500 \mathrm{~K}$. Different symbols are for different clusters, as noted in lower right. Lines shows the adopted temperature calibrations. Carbon and nitrogen are not shown because we cannot assume homogeneous abundances within clusters. 
Table 5

Internal Temperature Calibration Slopes

\begin{tabular}{lc}
\hline \hline Element & Slope Per $1000 \mathrm{~K}$ \\
\hline $\mathrm{C}$ & $\ldots$ \\
$\mathrm{N}$ & $\ldots$ \\
$\mathrm{O}$ & +0.007 \\
$\mathrm{Na}$ & -0.001 \\
$\mathrm{Mg}$ & -0.051 \\
$\mathrm{Al}$ & -0.046 \\
$\mathrm{Si}$ & -0.121 \\
$\mathrm{~S}$ & +0.060 \\
$\mathrm{~K}$ & -0.046 \\
$\mathrm{Ca}$ & +0.059 \\
$\mathrm{Ti}$ & -0.162 \\
$\mathrm{~V}$ & -0.177 \\
$\mathrm{Mn}$ & -0.206 \\
$\mathrm{Fe}$ & -0.055 \\
$\mathrm{Ni}$ & -0.124 \\
{$[\mathrm{M} / \mathrm{H}]$} & -0.056 \\
{$[\alpha / \mathrm{M}]$} & -0.028 \\
\hline
\end{tabular}

the observed scatter is less than 0.1 dex, and for many elements, it is below 0.05 dex. Generally, the errors increase with increasing temperature, decreasing metallicity, and decreasing S/N.

These empirical errors, which are distributed in the APOGEE data release (along with the raw FERRE errors), capture the scatter in elemental abundances observed in clusters over a narrow range of effective temperature. Over a broader range of temperature, they do not capture the degree to which our simple, metallicity-independent, linear temperature calibration relation fits the data. Since the observed stars in any given cluster do not usually span a large temperature range, we estimate the overall uncertainty in abundances, across a broad range of temperature, by measuring the scatter around the calibration relations of Figure 5. This is presented in the final column of Table 6 . These "global" uncertainties do not capture the likely dependence of the precision on temperature, metallicity, and signal-to-noise. They represent a more conservative estimate of the internal uncertainties.

The scatter within clusters indicates that the precision of our abundances is less than $0.1 \mathrm{dex}$ for all elements, with the possible exception of vanadium. For some abundances, especially if considering the sample over a restricted temperature range and high $\mathrm{S} / \mathrm{N}$, the precision can be better than 0.05 dex.

\subsubsection{External Calibration}

Table 4 gives the adopted literature values for $[\mathrm{Fe} / \mathrm{H}]$, along with references, for the calibration cluster sample. The difference between the derived ASPCAP values and the literature values for both the parameter $[\mathrm{M} / \mathrm{H}]$ and the abundance $[\mathrm{Fe} / \mathrm{H}]$ are shown in Figure 6 . We adopt the literature $[\mathrm{Fe} / \mathrm{H}]$ as a reference for our $[\mathrm{M} / \mathrm{H}]$ values because our method allows for variations in $\alpha$ elements, carbon, and nitrogen separately; implicit in this comparison is the assumption that the $\mathrm{Fe}$ abundance drives the overall metallicity in the APOGEE spectra.

The derived ASPCAP $[\mathrm{M} / \mathrm{H}]$ and $[\mathrm{Fe} / \mathrm{H}]$ are similar to the literature values near solar metallicity, but are $\sim 0.2-0.3 \mathrm{dex}$ more metal-rich than the literature for very metal-poor clusters. This is similar to what was seen for the DR10 data release (Mészáros et al. 2013). We adopted a quadratic relation in metallicity to derive calibration relations: ${ }^{32}$

$$
\begin{aligned}
{[\mathrm{M} / \mathrm{H}]_{\text {corr }}=} & {[\mathrm{M} / \mathrm{H}]_{\text {raw }}+0.0260 } \\
& +0.255[\mathrm{M} / \mathrm{H}]_{\text {raw }}+0.062[\mathrm{M} / \mathrm{H}]_{\text {raw }}^{2} \\
{[\mathrm{Fe} / \mathrm{H}]_{\text {corr }}=} & {[\mathrm{Fe} / \mathrm{H}]_{\text {raw }}+0.0326 } \\
& +0.245[\mathrm{M} / \mathrm{H}]_{\text {raw }}-0.042[\mathrm{M} / \mathrm{H}]_{\text {raw }}^{2}
\end{aligned}
$$

The scatter around this relation is 0.12 dex.

We have chosen to apply the calibration above to the $[\mathrm{M} / \mathrm{H}]$ parameter, but not to the $[\mathrm{Fe} / \mathrm{H}]$ abundances, given the impossibility of calibrating all of the elemental abundances in a homogeneous fashion. Most users will prefer to work with $[\mathrm{X} / \mathrm{Fe}]$ by subtracting the $[\mathrm{Fe} / \mathrm{H}]$ from $[\mathrm{X} / \mathrm{H}]$, and in many cases systematic errors in $[\mathrm{Fe} / \mathrm{H}]$ and $[\mathrm{X} / \mathrm{H}]$ will partly cancel out. If we were to apply the external calibration to $[\mathrm{Fe} / \mathrm{H}]$, it would have the effect of increasing the $[\mathrm{X} / \mathrm{Fe}]$ ratios of all elements toward lower metallicity. Since we do not know the source of the $[\mathrm{Fe} / \mathrm{H}]$ discrepancy at low metallicity, it is difficult to judge whether it is likely to affect all elements similarly, or whether it is specific to iron. As a result, we choose to leave all of the $[\mathrm{X} / \mathrm{Fe}]$ ratios on the native, consistent, ASPCAP scale.

The main issue addressed by the calibration relation is at the metal-poor end. If one were to ignore all clusters with $[\mathrm{Fe} / \mathrm{H}]<-0.5$, the remaining metal-rich clusters show little evidence for an offset or a trend with metallicity. However, the simple quadratic form of the relation adopted to address the issues at $[\mathrm{Fe} / \mathrm{H}]<-0.5$ results in a relation that changes by almost $0.3 \mathrm{dex}$ at $-0.5<[\mathrm{Fe} / \mathrm{H}]<0.5$. As a result, there are non-negligible differences between the calibrated and uncalibrated $[\mathrm{M} / \mathrm{H}]$ in this regime, and the uncalibrated value may be just as good as the calibrated ones for clusters with $[\mathrm{M} / \mathrm{H}]>-0.5$.

\subsection{The Abundance Scale}

The ASPCAP abundances are presented in the DR12 database as relative to hydrogen in the usual bracket notation:

$$
[\mathrm{X} / \mathrm{H}]=\log \left(n_{\mathrm{X}} / n_{\mathrm{H}}\right)-\log \left(n_{\mathrm{X}} / n_{\mathrm{H}}\right)_{\odot}
$$

where $n_{\mathrm{X}}$ and $n_{\mathrm{H}}$ are the number density of nuclei of element $\mathrm{X}$ and hydrogen, respectively. But some caveats apply. The abundances are not truly differential to the values that APOGEE derives for the Sun. Solar abundances are adopted from Asplund et al. (2005) and used for computing model atmospheres (Mészáros et al. 2012) and fine-tuning the line list (M. Shetrone et al. 2015, in preparation). The line list used for spectral synthesis includes, in addition to laboratory and theoretical transition probabilities and damping constants, modifications to provide a compromise match to both the solar spectrum and Arcturus. However, only atomic lines were adjusted, and even for those, only within limits.

Therefore, the ASPCAP abundances are not strictly differential to those of the Sun. Given the way the line lists were determined, the existence of the temperature trends in the raw abundances, and the lack of external calibration (apart from

\footnotetext{
32 We note that the relations presented here, which were used for the DR12 release, were derived using a literature metallicity of 0.04 for NGC 2158, instead of the more recent literature value of -0.28 presented here and used in Figure 6.
} 
Table 6

Empirical Uncertainty Relation Coefficients and Values

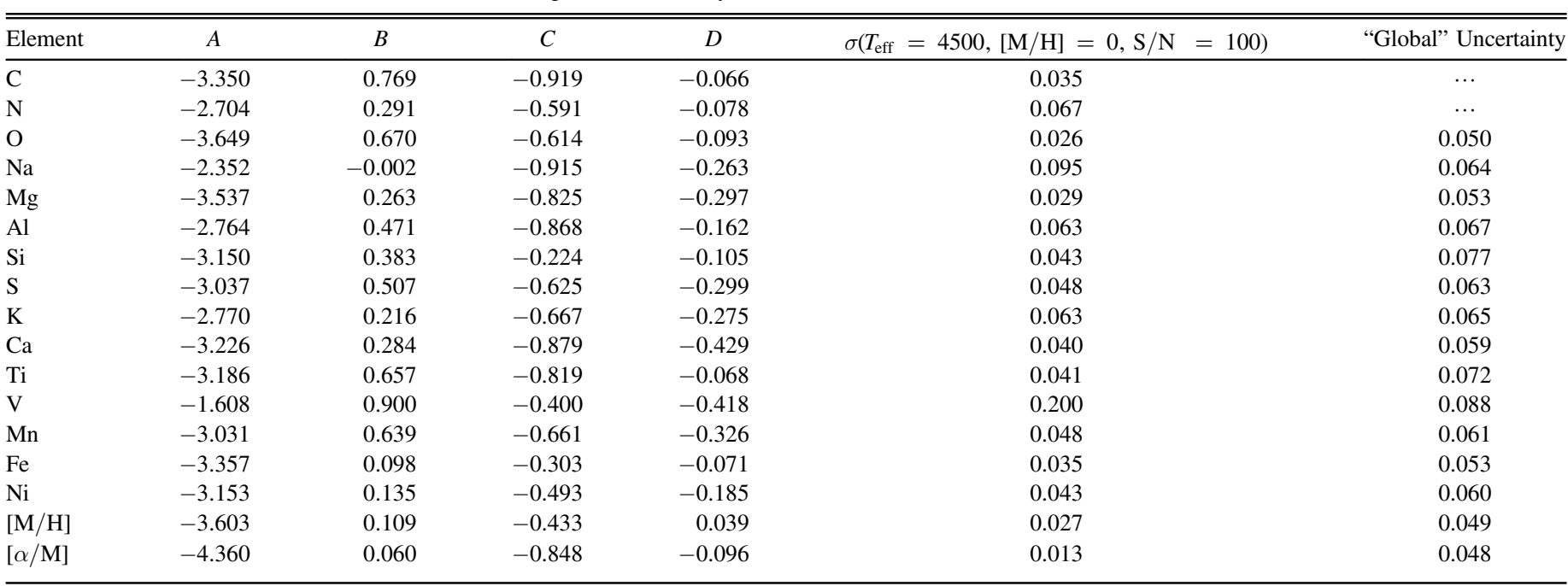
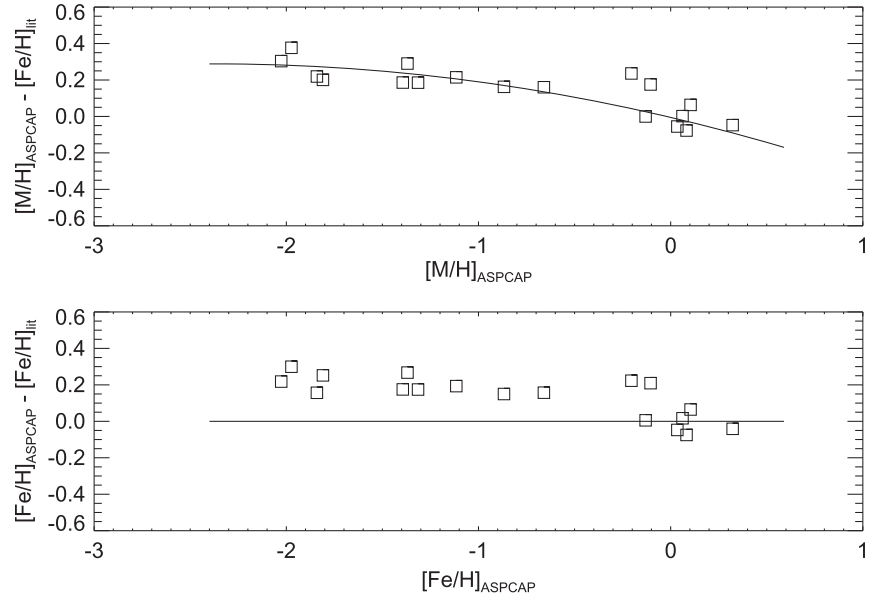

Figure 6. External elemental abundance calibration.

that for $[\mathrm{M} / \mathrm{H}])$, APOGEE defines its own relative abundance scale. We consider the accuracy of this scale by several external comparisons, including observations of the solar spectrum and of Arcturus, observations of stars with independently measured abundances, and consideration of the locus in paramters and abundances of the entire APOGEE sample.

\subsubsection{Solar and Arcturus Abundances}

Observations of the solar spectrum, obtained by observing the asteroid Vesta, and of Arcturus were made using the NMSU $1 \mathrm{~m}$ feed to the APOGEE spectrograph. These spectra are of particular interest because their abundances set the standard for many abundance studies, they have high resolution spectral atlases, and because the combination of the abundances and high resolution spectra were used to tune the APOGEE line list.

However, the internal calibration that depends on the ASPCAP temperature was derived from the cluster sample which is significantly cooler than the Sun. Additionally, the calibration was derived from giants, not dwarfs, so the applicability of the calibration to the solar abundances is debatable, and this complicates the solar comparison. Table 7 presents the solar abundances derived from the Vesta spectrum, showing both the uncalibrated abundances and the calibrated
Table 7

Solar Parameters and Abundances from Vesta

\begin{tabular}{lcc}
\hline \hline Element & Uncalibrated ASPCAP & Calibrated ASPCAP \\
\hline$T_{\text {eff }}$ & $5728 . \pm 91.5$ & $5812 . \pm 91.5$ \\
$\log g$ & $4.54 \pm 0.11$ & $4.49 \pm 0.11$ \\
{$[\mathrm{M} / \mathrm{H}]$} & $0.03 \pm 0.03$ & $0.11 \pm 0.03$ \\
{$[\alpha / \mathrm{M}]$} & $0.00 \pm 0.01$ & $0.02 \pm 0.01$ \\
{$[\mathrm{C} / \mathrm{H}]$} & $0.06 \pm 0.07$ & $0.06 \pm 0.07$ \\
{$[\mathrm{~N} / \mathrm{H}]$} & $0.10 \pm 0.06$ & $0.10 \pm 0.06$ \\
{$[\mathrm{O} / \mathrm{H}]$} & $0.03 \pm 0.01$ & $0.02 \pm 0.01$ \\
{$[\mathrm{Na} / \mathrm{H}]$} & $0.03 \pm 0.03$ & $0.03 \pm 0.03$ \\
{$[\mathrm{Mg} / \mathrm{H}]$} & $0.07 \pm 0.03$ & $0.11 \pm 0.03$ \\
{$[\mathrm{Al} / \mathrm{H}]$} & $-0.03 \pm 0.01$ & $0.00 \pm 0.01$ \\
{$[\mathrm{Si} / \mathrm{H}]$} & $0.03 \pm 0.03$ & $0.12 \pm 0.03$ \\
{$[\mathrm{~S} / \mathrm{H}]$} & $0.26 \pm 0.04$ & $0.22 \pm 0.04$ \\
{$[\mathrm{~K} / \mathrm{H}]$} & $-0.07 \pm 0.03$ & $-0.04 \pm 0.03$ \\
{$[\mathrm{Ca} / \mathrm{H}]$} & $0.02 \pm 0.07$ & $-0.03 \pm 0.07$ \\
{$[\mathrm{Ti} / \mathrm{H}]$} & $-0.07 \pm 0.04$ & $0.05 \pm 0.04$ \\
{$[\mathrm{~V} / \mathrm{H}]$} & $0.11 \pm 0.05$ & $0.24 \pm 0.05$ \\
{$[\mathrm{Mn} / \mathrm{H}]$} & $0.04 \pm 0.03$ & $0.19 \pm 0.03$ \\
{$[\mathrm{Fe} / \mathrm{H}]$} & $0.01 \pm 0.07$ & $0.05 \pm 0.07$ \\
{$[\mathrm{Ni} / \mathrm{H}]$} & $0.01 \pm 0.15$ & $0.10 \pm 0.15$ \\
\hline
\end{tabular}

abundances determined by applying the internal calibration correction appropriate for $5250 \mathrm{~K}$, which is the high temperature end of the calibration sample.

In general, the observed uncalibrated solar abundances are within the expected errors, except for magnesium, sulfur, and vanadium. Applying the calibration (again, outside of its derived range) slightly improves some of the abundances, but makes the magnesium, silicon, manganese, and vanadium abundances worse. Systematic offsets from the solar abundance all appear to be within 0.1 apart from sulfur and vanadium, and perhaps silicon (if one considers the calibrated value).

Table 8 presents the ASPCAP Arcturus abundances, along with several different comparison values: the abundances that were adopted to tune the line list, a set of abundances derived from a high-resolution near-IR FTS spectrum by Smith et al. (2013) using a similar (but not identical) linelist to the current APOGEE linelist, and a set of abundances derived from atomic 
Table 8

Arcturus Parameters and Abundances

\begin{tabular}{|c|c|c|c|c|c|c|c|c|}
\hline Element & ASPCAP & Adopted & Smith & Ramirez & ASPCAP $[\mathrm{X} / \mathrm{Fe}]$ & Adopted $[\mathrm{X} / \mathrm{Fe}]$ & Smith $[\mathrm{X} / \mathrm{Fe}]$ & Ramirez $[\mathrm{X} / \mathrm{Fe}]$ \\
\hline $\log g$ & $1.71 \pm 0.11$ & $\cdots$ & 2.10 & 1.66 & $\cdots$ & $\ldots$ & $\ldots$ & $\ldots$ \\
\hline$[\mathrm{M} / \mathrm{H}]$ & $-0.64 \pm 0.03$ & $\ldots$ & -0.40 & $\ldots$ & $\ldots$ & $\ldots$ & $\ldots$ & $\ldots$ \\
\hline$[\alpha / \mathbf{M}]$ & $0.20 \pm 0.02$ & $\ldots$ & 0.40 & $\ldots$ & $\ldots$ & $\ldots$ & $\ldots$ & $\ldots$ \\
\hline$[\mathrm{C} / \mathrm{H}]$ & $-0.43 \pm 0.04$ & -0.43 & -0.43 & -0.09 & 0.15 & 0.09 & 0.04 & 0.43 \\
\hline$[\mathrm{N} / \mathrm{H}]$ & $-0.52 \pm 0.08$ & -0.14 & -0.14 & $\ldots$ & 0.05 & 0.38 & 0.33 & $\ldots$ \\
\hline$[\mathrm{Mg} / \mathrm{H}]$ & $-0.40 \pm 0.03$ & -0.12 & -0.38 & -0.15 & 0.18 & 0.40 & 0.09 & 0.37 \\
\hline$[\mathrm{Al} / \mathrm{H}]$ & $-0.32 \pm 0.07$ & -0.22 & -0.21 & -0.18 & 0.26 & 0.30 & 0.26 & 0.34 \\
\hline$[\mathrm{Si} / \mathrm{H}]$ & $-0.33 \pm 0.04$ & -0.12 & -0.39 & -0.19 & 0.25 & 0.40 & 0.08 & 0.33 \\
\hline$[\mathrm{S} / \mathrm{H}]$ & $-0.41 \pm 0.04$ & -0.12 & $\ldots$ & $\ldots$ & 0.17 & 0.40 & $\ldots$ & $\ldots$ \\
\hline$[\mathrm{K} / \mathrm{H}]$ & $-0.54 \pm 0.06$ & -0.52 & -0.29 & -0.32 & 0.04 & 0.00 & 0.18 & 0.20 \\
\hline$[\mathrm{Ca} / \mathrm{H}]$ & $-0.51 \pm 0.04$ & -0.42 & -0.47 & -0.41 & 0.07 & 0.10 & 0.00 & 0.11 \\
\hline$[\mathrm{Ni} / \mathrm{H}]$ & $-0.51 \pm 0.04$ & -0.52 & -0.46 & -0.46 & 0.07 & 0.00 & 0.01 & 0.06 \\
\hline
\end{tabular}

transitions in the optical spectrum by Ramírez \& Allende Prieto (2011).

Assessing the quality of the APOGEE abundances is challenging because there is not a consensus in the literature for all Arcturus abundances. Note that we do not necessarily expect the ASPCAP results to match the abundances that were adopted to tune the line list, because the line list tuning involved making compromises between the solar spectrum and the Arcturus spectrum (with the latter receiving lower weight, M. Shetrone et al. 2015, in preparation) and only allowed atomic transition probabilities to vary within limits.

Several different potential issues can be noted. For carbon, the near-IR abundances agree, but differ significantly from the Ramírez \& Allende Prieto (2011) value; however, Sneden et al. (2014) find a value much closer to the near-IR values. For oxygen, the ASPCAP value differs significantly from either the alternate near-IR or the optical abundance, and seems quite low given that Arcturus has generally been found to be enhanced in $\alpha$-element abundances. This may partly be understood because oxygen is primarily derived from $\mathrm{OH}$ lines, which are quite sensitive to temperature (see Smith et al. 2013) and the raw Arcturus ASPCAP temperature $(4207 \mathrm{~K})$ is lower than the literature values (here is a case where one might have gotten better results if the calibrated temperatures were used for the abundances). For other $\alpha$ elements (Mg, Si, Ca), the ASPCAP values agree fairly well with the Smith et al. (2013) values, but are lower than the Ramírez \& Allende Prieto (2011) values, suggesting less overall $\alpha$ enhancement.

\subsubsection{Other Individual Star Comparisons}

In addition to Arcturus and Vesta, we obtained NMSU $1 \mathrm{~m}$ + APOGEE observations of several other bright stars ( $\delta$ Oph, $\beta$ And, $\mu$ Leo) for which Smith et al. (2013) have derived abundances from high-resolution near-IR spectra obtained with an FTS spectrograph and using the ASPCAP line list. We also have observations of a number of nearby stars with published abundances from optical analyses by Reddy et al. (2003, 2006), and Bensby et al. (2014); because these stars are bright, observations were also all obtained with the NMSU $1 \mathrm{~m}+$

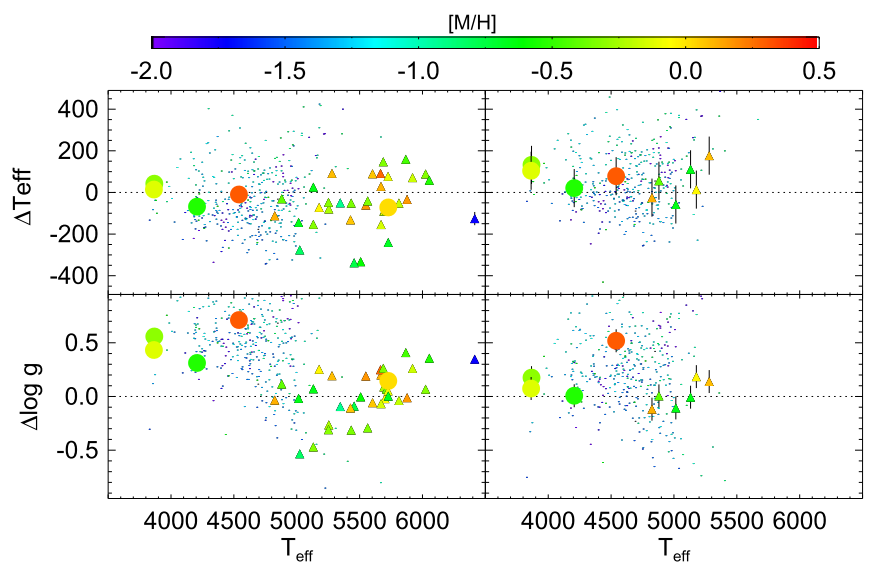

Figure 7. Comparison with literature spectroscopic effective temperatures and surface gravities. Left panel is uncalibrated ASPCAP results; right is for calibrated results. Differences are ASPCAP minus reference values. Large circles are parameters from FTS spectra (Smith et al. 2013), filled triangles from optical spectra and analysis, and small points from globular cluster analysis of Mészáros et al. (2015).

APOGEE. However, these are mostly warmer stars with higher surface gravity and fall outside the range of most APOGEE main sample stars and thus outside the range where we have internally calibrated the abundances.

Mészáros et al. (2015) have derived abundances from APOGEE spectra for a number of these globular cluster members. In addition, Cunha et al. (2015) have derived $\mathrm{Na}, \mathrm{O}$ and $\mathrm{Fe}$ abundances from manual analysis (i.e., doing synthesis around individual lines) of APOGEE spectra for stars in the cluster NGC 6791, so these provide an opportunity to test the APOGEE/ASPCAP abundances against those derived by more traditional methods from the same spectra. However, these independent analyses of the APOGEE spectra were done using a slightly different version of the APOGEE linelist, so exact agreement is not expected (but the changes should be small, apart from K).

Results for all of these samples are shown in Figures 7-10 for stellar parameters $\left(T_{\text {eff }}\right.$ and $\left.\log g\right), \mathrm{CNO}, \alpha$-element, and 
$[\mathrm{M} / \mathrm{H}]$

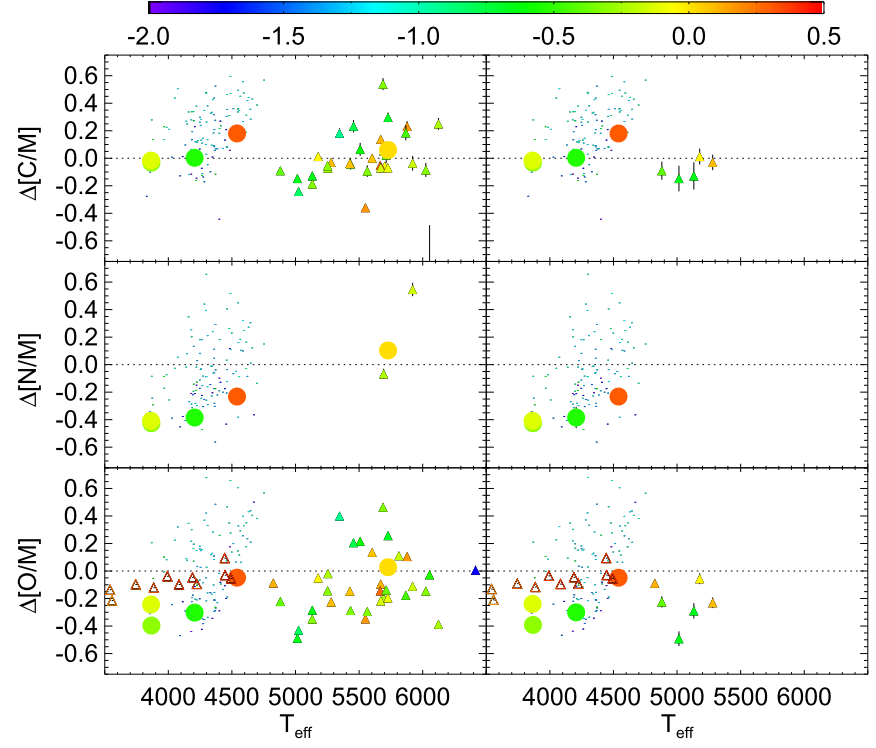

Figure 8. Comparison with literature abundances for $\mathrm{CNO}$ elements. Left panel is uncalibrated ASPCAP results; right is for calibrated results. Differences are ASPCAP minus reference values. Large circles are abundances from FTS spectra (Smith et al. 2013), filled triangles from optical spectra and analysis, open triangles from independent analysis of APOGEE spectra (Cunha et al. 2015), and small points from Mészáros et al. (2015). The empirical uncertainties as discussed in the text are shown as error bars, but these are often comparable or smaller than the size of the points.

other elements, respectively. In these plots, uncalibrated parameters/abundances are shown in the left panels, and calibrated parameters/abundances are shown on the right, both plotted as a function of effective temperature. Since, as discussed above, stars with higher surface gravity are not calibrated, most of the warmer stars are missing for the calibrated parameters/abundances. In all plots, the points are color-coded by the derived overall metallicity, with blue representing metal-poor stars and red metal-rich ones. Different symbols represent different comparison samples: solid circles are the results from the FTS spectra, solid triangles from the optical analyses of solar neighborhood stars. Open triangles show the independent analysis for NGC 6791, and the small dots show the results for the independent analysis of globular cluster stars. Although it is challenging to interpret all of the comparisons because the comparison samples are heterogeneous and each have their own associated uncertainties, a few potential issues can be noticed.

From Figure 8, the ASPCAP carbon abundances for giants seem to be in reasonable agreement with literature values. ASPCAP nitrogen abundances seem to be significantly lower, and oxygen abundances moderately lower.

The $\alpha$ element abundances in Figure 9 suggest that $\mathrm{Mg}$ compares fairly well, that $\mathrm{Si}$ is a bit high in the ASPCAP abundances, and that $\mathrm{Ca}$ and $\mathrm{Ti}$ are a bit low. Titanium results may indicate a trend with temperature; as discussed below, there is other indication that ASPCAP titanium abundances may have significant issues that are not currently fully understood.

The results for $\mathrm{Al}, \mathrm{Fe}$, and $\mathrm{Ni}$, shown in Figure 10, are encouraging. The offset for $\mathrm{K}$ seen in that Figure from the analysis of the FTS spectra by Smith et al. (2013) can be partly

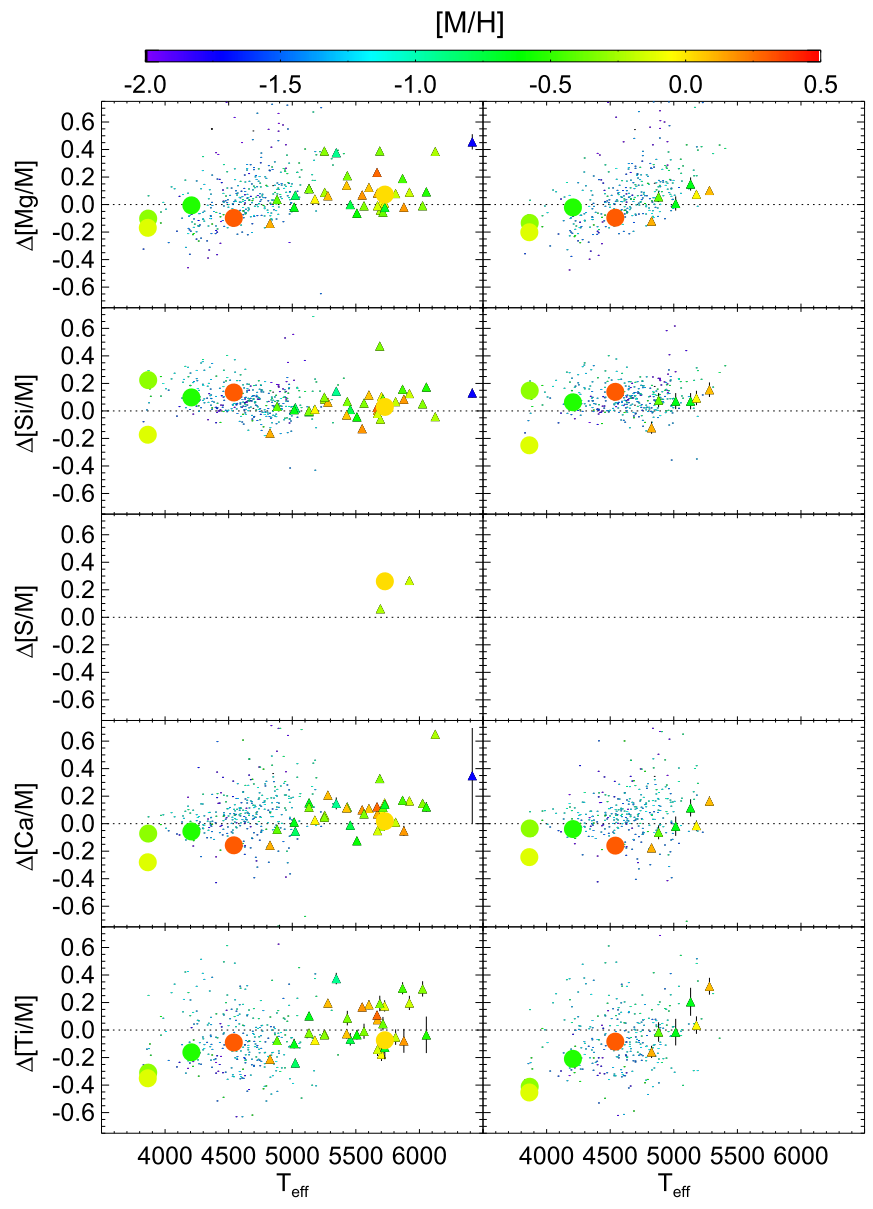

Figure 9. Same as Figure 8 for $\alpha$-element abundances ( $\mathrm{Mg}, \mathrm{Si}, \mathrm{S}, \mathrm{Ca}, \mathrm{Ti})$.

understood by noting that significantly different $\log g f$ values were used for the $\mathrm{K}$ lines in that study.

For the metal-poor globular cluster stars, the ASPCAP temperatures, surface gravities, and abundances are offset from the literature values and, in fact, even from the values measured from the independent analyses of the APOGEE spectra. We suspect that this is related to non-standard abundance patterns in some of the globular cluster stars, e.g., oxygen abundances that do not scale with other $\alpha$ elements. The assumption that $\alpha$ elements vary in lockstep, made during the initial stellar parameter determination, may lead to stellar parameters that are systematically offset, which is evident in the comparison of effective temperatures for the globular cluster stars. The systematic offsets with the stellar parameters may then lead to systematic offsets in some of the individual abundances. In addition, since features such as $\mathrm{CN}$ and $\mathrm{CO}$ can be very weak in metal-poor stars, the adopted scheme of allowing $[\mathrm{C} / \mathrm{M}]$ and $[\mathrm{N} / \mathrm{M}]$ to vary with the stellar parameters may cause problems.

Overall, these comparisons suggest that there may be nonnegligble systematic offsets between ASPCAP abundances and literature abundances for some elements. Such offsets are perhaps not uncommon in abundance analysis where derived values have not been adjusted relative to those obtained from a source of similar stellar parameters (e.g., the Sun for analysis of warmer dwarfs). Nonetheless, they do indicate that, in an external sense, the accuracy of ASPCAP abundances may be poorer $(0.1-0.2 \mathrm{dex})$ than the internal precision. 


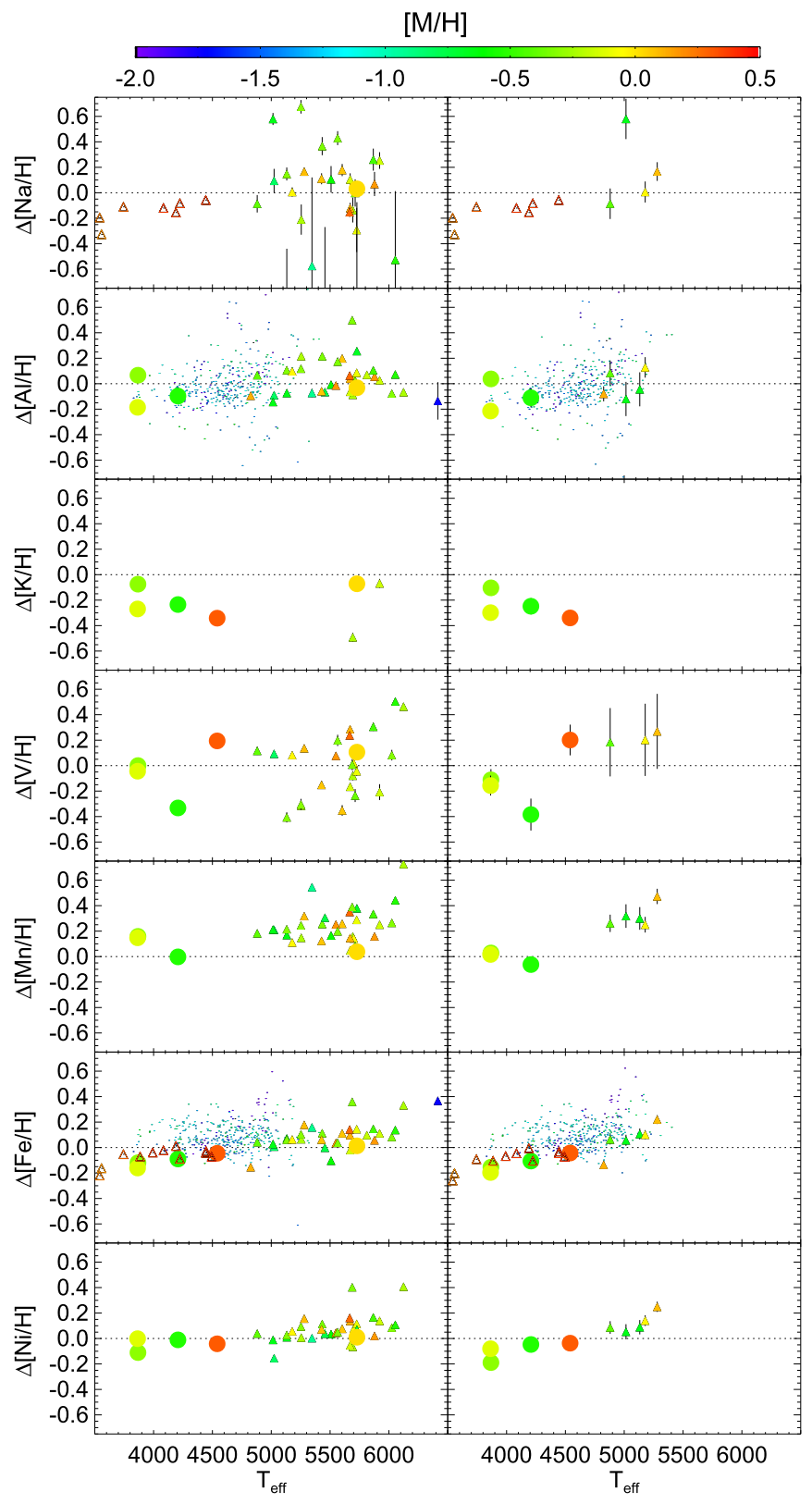

Figure 10. Same as Figure 8 for $\mathrm{Na}, \mathrm{Al}, \mathrm{K}, \mathrm{V}, \mathrm{Mn}, \mathrm{Fe}$, and $\mathrm{Ni}$.

\subsection{Sample Characteristics}

In this section, we provide information about the global distribution of properties across the entire APOGEE sample, to help to indicate the quality of derived parameters and abundances across the large parameter space of the APOGEE stars.

\subsubsection{Quality of the Fits}

For each stellar spectrum that is fit by ASPCAP, FERRE returns a $\chi^{2}$ of the fit. The $\chi^{2}$ distribution for the entire calibrated APOGEE DR12 sample is plotted in Figure 11. It is clear that the quality of the fits is poorer for cooler stars. This is perhaps not unexpected as the number and depth of spectral features generally increase at cooler temperatures, so any problems, e.g., with the line list, will be amplified at cooler

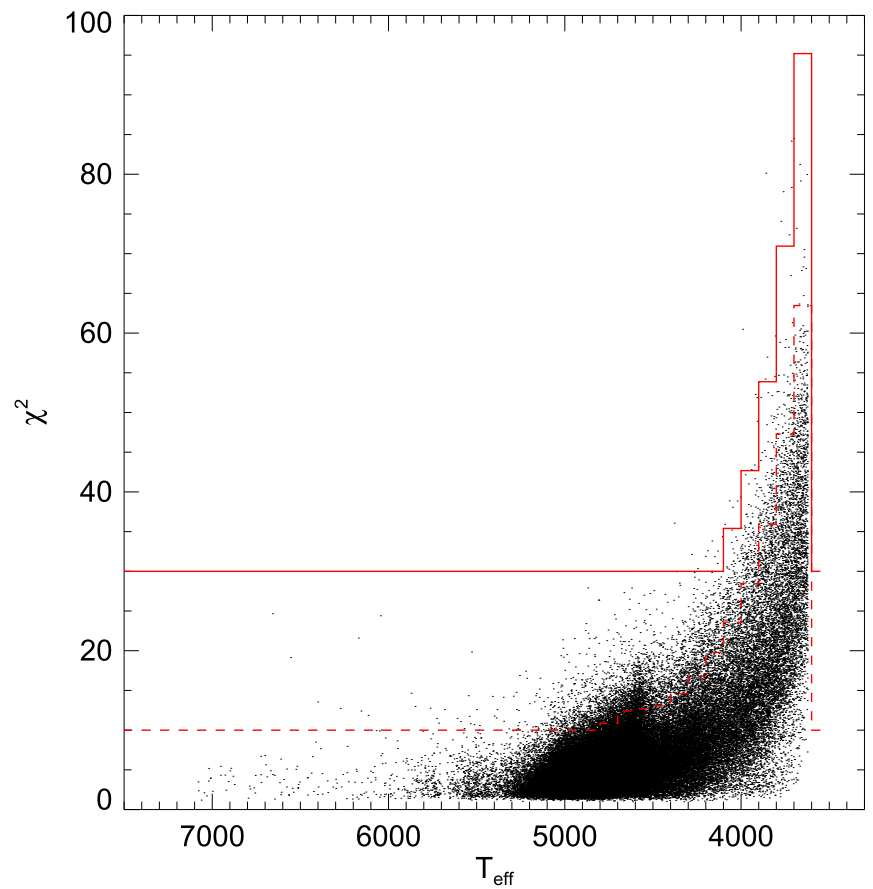

Figure 11. $\chi^{2}$ distribution for full APOGEE giant sample. Lines show the limits above which the CHI2_WARN (dashed) and CHI2_BAD (solid) bits are set.

temperatures. These results suggest that there is probably more uncertainty in the ASPCAP results at cooler temperatures.

For each star, if the $\chi^{2}$ value is larger than the typical value at the temperature of the star, a bit is set in the ASPCAPFLAG (see Section 7.1.3): the dashed and solid lines in Figure 11 show the levels above which the CHI2_WARN and CHI2_BAD bits are set, respectively.

\subsubsection{Stellar Parameters}

One way of testing the quality of the derived stellar parameters is to see to what extent we recover the expected trends on an HR diagram. Figure 12 presents an HR diagram from both the uncalibrated APOGEE DR12 sample (top) and the calibrated sample (bottom), with points color-coded by their derived metallicity, $[\mathrm{M} / \mathrm{H}]$. We also overlay isochrones for a 4 Gyr old population (Bressan et al. 2012) for $[\mathrm{M} / \mathrm{H}]=-1.8$, $-1.5,-1.0,-0.5,0 ., 0.3$.

The uncalibrated results (top panel) show that the dwarfs, while clearly separated from the giants, have stellar parameters that are offset from the stellar models. In part, this is probably due to the exclusion of a stellar rotation dimension in the synthetic grid, but since not all stars are expected to have significant rotation, there are probably additional effects as well. While the ASPCAP parameters are of sufficient quality to separate dwarfs from giants, these issues, along with the lack of calibrators available for stars of high surface gravity, lead us to recommend that the parameters and abundances for dwarfs be used only with caution, and no attempt has been made to calibrate them for the current data release.

The bottom panel of Figure 12 shows the calibrated giant sample. The calibrated parameters show the expected change in the locus of points as a function of metallicity. This agreement, which is obtained without imposing any assumptions about the relation between effective temperature, surface gravity, and 


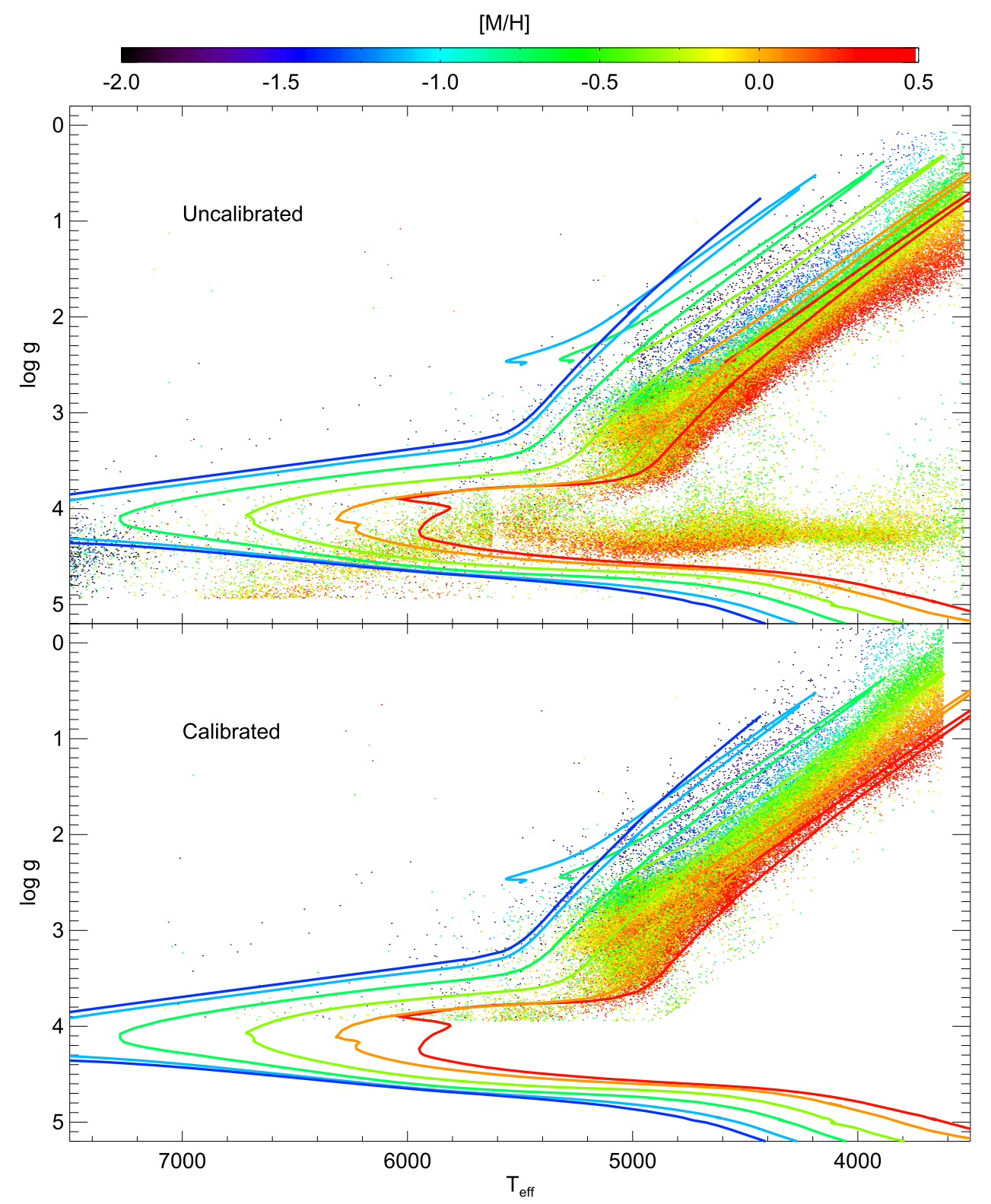

Figure 12. Effective temperature—surface gravity—metallicity distributions for uncalibrated full APOGEE sample (top) and for calibrated giant sample (bottom). Isochrones from Bressan et al. (2012) for a $4 \mathrm{Gyr}$ population, with metallicities $[\mathrm{M} / \mathrm{H}]=-1.8,-1.5,-1.0,-0.5,0 ., 0.3$ are overlaid.

metallicity, is quite encouraging. We note that some scatter in the locus at fixed metallicity is expected if there exists a range of ages at given metallicity; this full sample includes stars from across the Milky Way, so this is not expected to be a coeval sample.

Potential problems with the surface gravities for red clump stars are visible, as stellar evolution models predict that red clump stars should have near constant surface gravity, while the ASPCAP sequence slopes to higher surface gravity at higher temperature. While there is some possibility that standard stellar evolutionary models could have some issues, the ASPCAP surface gravities for RC stars are also higher than those determined from asteroseismic analysis, and the calibration we have applied, which was derived from RGB stars, is likely to make the shape of the RC sequence worse, as discussed in Section 5.2,

Figure 13 shows the $[\alpha / \mathrm{M}]$ versus $[\mathrm{M} / \mathrm{H}]$ relation for the calibrated giant sample, color coded by temperature. The expected trend toward higher $[\alpha / \mathrm{M}]$ at lower metallicity is apparent. However, the cooler stars (blue points) appear to deviate from the locus of stars of other temperatures. While it is possible that there are some astrophysical effects contributing here (cooler stars are more likely to be at larger distances), a more likely interpretation is that the $[\alpha / \mathrm{M}]$ results (and probably other abundances) are less reliable at the cooler end of the ASPCAP temperature range, i.e., $T<4000 \mathrm{~K}$. This is also the temperature range where the quality of the fits gets significantly worse. As a result, users should exercise caution when using results for the cooler stars.

\subsubsection{Stellar Abundances}

Figure 14 presents plots of $[\mathrm{X} / \mathrm{Fe}]$ versus $[\mathrm{Fe} / \mathrm{H}]$ for all 15 elements for a subsample of stars with high S/N (>200); the scatter in these plots increases for lower $\mathrm{S} / \mathrm{N}$. These are presented to assess to what degree some of the expected trends 


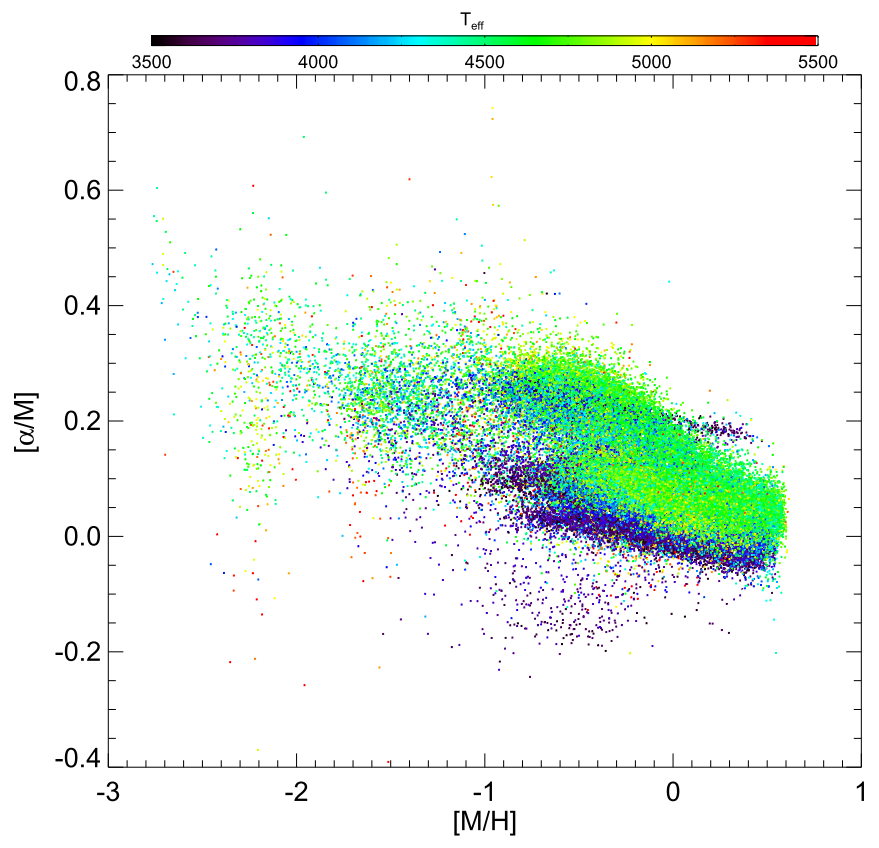

Figure 13. Metallicity- $[\alpha / \mathrm{M}]$-temperature relation for calibrated APOGEE giant sample.

are observed; a more extensive discussion of these plots in terms of chemical evolution is beyond the scope of this paper. Points in the plots are color-coded by effective temperature; black points are those for which the empirical uncertainties (Section 5.4.2) are larger than 0.1 dex.

Previous studies from near the solar neighborhood have shown, in general, an increasing fraction of stars with enhanced $[\alpha / \mathrm{Fe}]$ ratios at lower metallicity (with contributions from higher $[\alpha / \mathrm{Fe}]$ starting near or just below solar metallicity), while other elements tend to have less variation. The APOGEE results for the $\alpha$ elements $\mathrm{O}, \mathrm{Mg}, \mathrm{Si}, \mathrm{S}$, and $\mathrm{Ca}$ show trends of increasing abundance relative to $\mathrm{Fe}$ at lower metallicity, as expected, although the behavior of $\mathrm{Mg}$ at the lowest metallicities indicates some possible issues in that regime. However, Ti does not indicate any significant trend. Given that such a trend has been clearly observed for titanium in the solar neighborhood (e.g., Bensby et al. 2014), this, along with the individual star comparison discussed previously, suggests that there is some issue that may be affecting the reliability of the ASPCAP Ti abundance. Possible issues with the ASPCAP derived $\mathrm{Ti}$ abundances will be investigated further in future work.

While the shape of the $\alpha$ element locii are generally as expected, the location of the higher metallicity stars suggest possible problems with the overall accuracy (zeropoint) of the APOGEE/ASPCAP abundances. The $\mathrm{Si}$ and $\mathrm{S}$ abundances ratios $([\mathrm{Si} / \mathrm{Fe}]$ and $[\mathrm{S} / \mathrm{Fe}])$ are greater than zero at solar metallicity by $0.1-0.2$ dex, while the $\mathrm{Ca}$ abundance $([\mathrm{Ca} / \mathrm{Fe}])$ is around -0.1 . As noted above, some offsets are perhaps not unexpected since we do not reference our abundances to those of any well-calibrated object, and also since we correct small temperature trends of abundances to the arbitrary fiducial temperature of $4500 \mathrm{~K}$.

Results for $\mathrm{Na}$ and $\mathrm{V}$ show large scatter; features from these elements are weak and most likely the scatter results from limited precision of the APOGEE measurements.
The points in Figure 14 are color-coded by the derived effective temperature, so that remaining trends with temperature (after internal calibration) may be discerned. In general, it seems that temperature trends are relatively small, with the possible exception of those for $\mathrm{Ti}, \mathrm{Mn}$, and $\mathrm{Ni}$. However, given that cooler giants are more luminous, stars of different temperatures likely sample different regions of the Milky Way, so apparent abundance trends with temperature do not necessarily represent an issue with the abundance determinations, as the chemical relations may vary across the Milky Way.

Overall, these results support our previous assessment that the internal precision of APOGEE/ASPCAP abundances is fairly $\operatorname{good}(<0.1 \mathrm{dex})$, while the external accuracy is more uncertain, with offsets of $0.1-0.2$ dex possible in some elements. Some elements appear to give tighter chemical relations than other elements, but this is perhaps not atypical for abundance analysis; in different wavelength regimes, different elements have different numbers of lines with different strengths, and some lines may be more affected by conditions such as non-LTE that we are not modeling.

\subsection{Persistence}

As noted in the description of the instrument, portions of two of the SDSS-III/APOGEE detectors have strong persistence that affects roughly one-third of the fibers over $40 \%$ of the APOGEE spectral range. Because the effect is complicated, not fully understood, and depends on the details of the exposure history, no attempt has yet been made to correct for it in SDSSIII/APOGEE analysis.

We attempt to determine whether persistence has a significant effect on the derived stellar parameters and abundances by comparing results for objects whose spectra were recorded outside the persistence region in all visits against results for objects whose spectra were recorded within the highest persistence region in all visits; there are some intermediate cases where some visits are recorded in the persistence region, and some outside of it (since plates are generally replugged between visits), as well as cases where spectra fall on regions of intermediate or lower persistence.

Results are shown in Figures 15-18. In all cases, we show only fainter stars with $H>11$, which tend to be more affected by persistence than brighter stars. The left plots show the results for stars that do not fall in the persistence region for any of the visits, and the right plots show results for stars that fall in the high persistence region in all visits. Figure 15 suggests that persistence does not seem to impact the parameters dramatically. However, for the individual elemental abundances, persistence can have a noticeable effect. In particular, the relations for $\mathrm{N}, \mathrm{Mg}, \mathrm{S}, \mathrm{K}, \mathrm{Ca}, \mathrm{Ti}, \mathrm{Mn}$, and $\mathrm{Ni}$ show significantly larger scatter or offsets for stars that fall in the high persistence region; given that some of these have features that are not within the persistence region, the stellar parameters must be affected at some level.

As a result, users are cautioned about the use of some of the individual element abundances for stars that were observed within the persistence region. These stars can be identified using the star quality bitmask described below.

\section{DATA PRODUCTS AND ACCESS}

APOGEE data can be accessed in several different ways. 


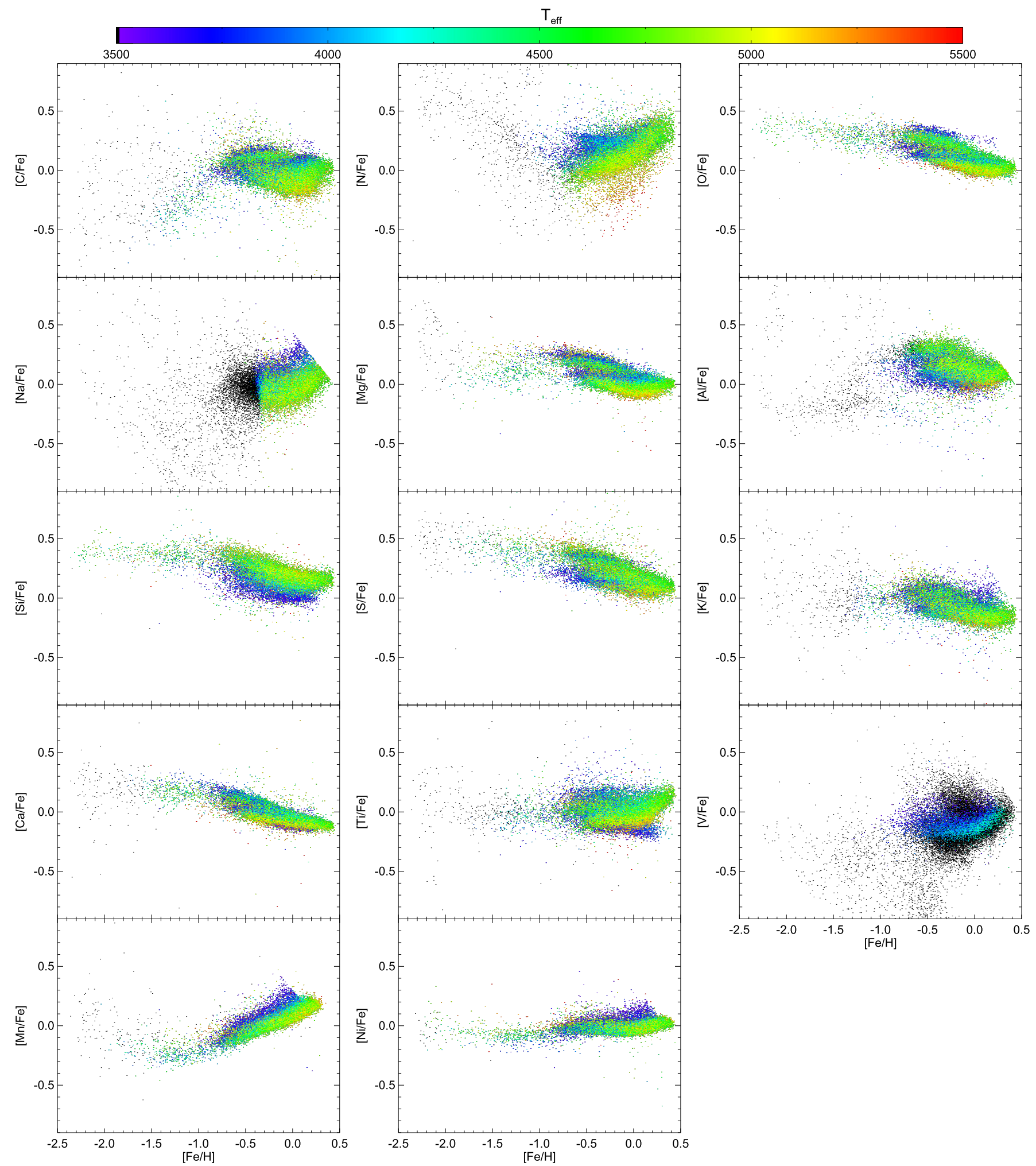

Figure 14. Abundance ratio plots for high $\mathrm{S} / \mathrm{N}(>200)$ stars from full APOGEE giant sample. Points are color coded by effective temperature; black points are stars with large uncertainties (empirical uncertainties in $[\mathrm{X} / \mathrm{Fe}]$ greater than $0.1 \mathrm{dex}$ ).

\subsection{Catalog Archive Server (CAS)}

Catalog data are also stored in a database called the CAS, which can be accessed at http://skyserver.sdss3.org/dr12. This database contains catalog information derived from the spectra, but not the spectra themselves.
The CAS contains a number of ways of interacting with the database, including low-level SQL access through the CasJobs interface at skyserver.sdss3.org/Casjobs. More information is available at skyserver.sdss3.org.

The CAS provides access to several database tables: 


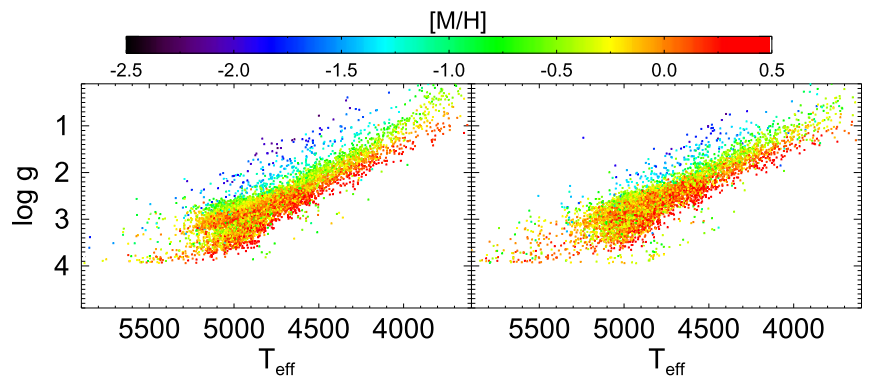

Figure 15. Persistence comparison for $T_{\text {eff }}$ vs. $\log g$ for stars with $H>11$. Left panel has stars with all observations outside of persistence region, while right panel has stars with all observations inside of persistence region. Points are color-coded by temperature.

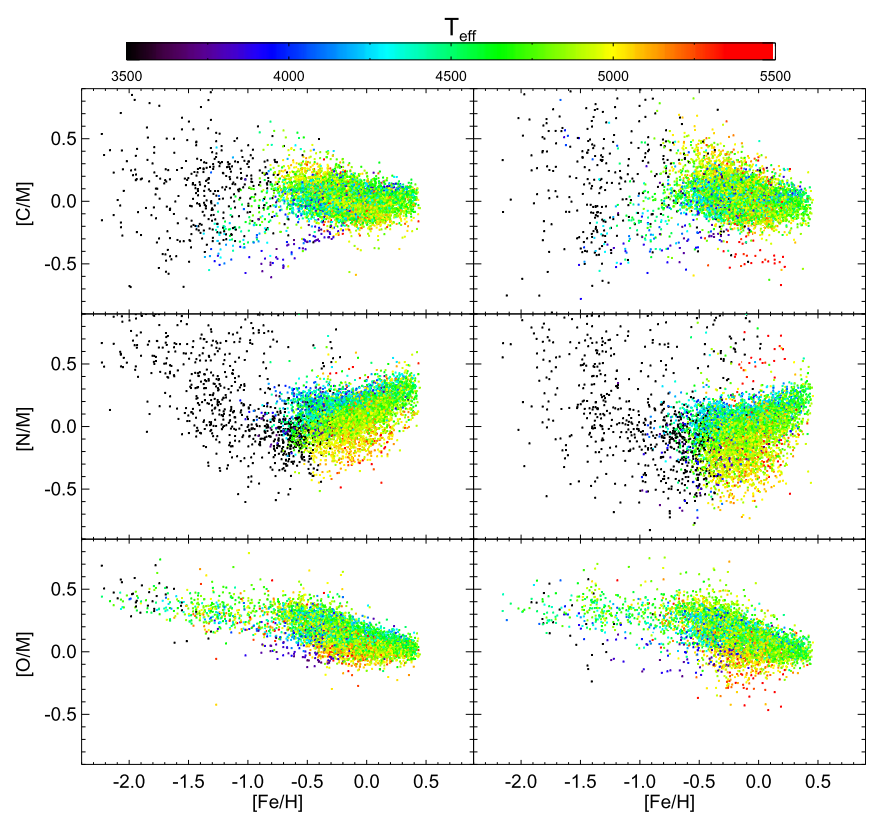

Figure 16. Persistence comparison for $\mathrm{CNO}$ abundances for stars with $H>11$. Left panel has stars with all observations outside of persistence region, while right panel has stars with all observations inside of persistence region. Points are color-coded by temperature; black points have empirical uncertainties greater than 0.1 dex.

1. apogeeStar-a table with basic information for each combined spectrum, including targeting flags, radial velocity information, and the STARFLAG and ANDFLAG data quality bitmasks for each individual star;

2. aspcapStar-a table with the ASPCAP parameters, abundances, uncertainties, and bitmasks flagging possible conditions with the parameters and abundances;

3. apogeeVisit-a table with the radial velocity information for each individual visit of each star;

4. apogeeObject- a table with all of the ancillary catalog/ targeting information for the parent sample of stars in each field from which the observed targets were drawn;

5. apogeePlate - a table with information about each plate observed;

6. apogeeDesign - a table that contains information about each plate design;

7. apogeeField - a table with information about each field that was observed, which includes the LOCATION_ID numeric value that is used to index different fields, and also the planned number of visits for each field.

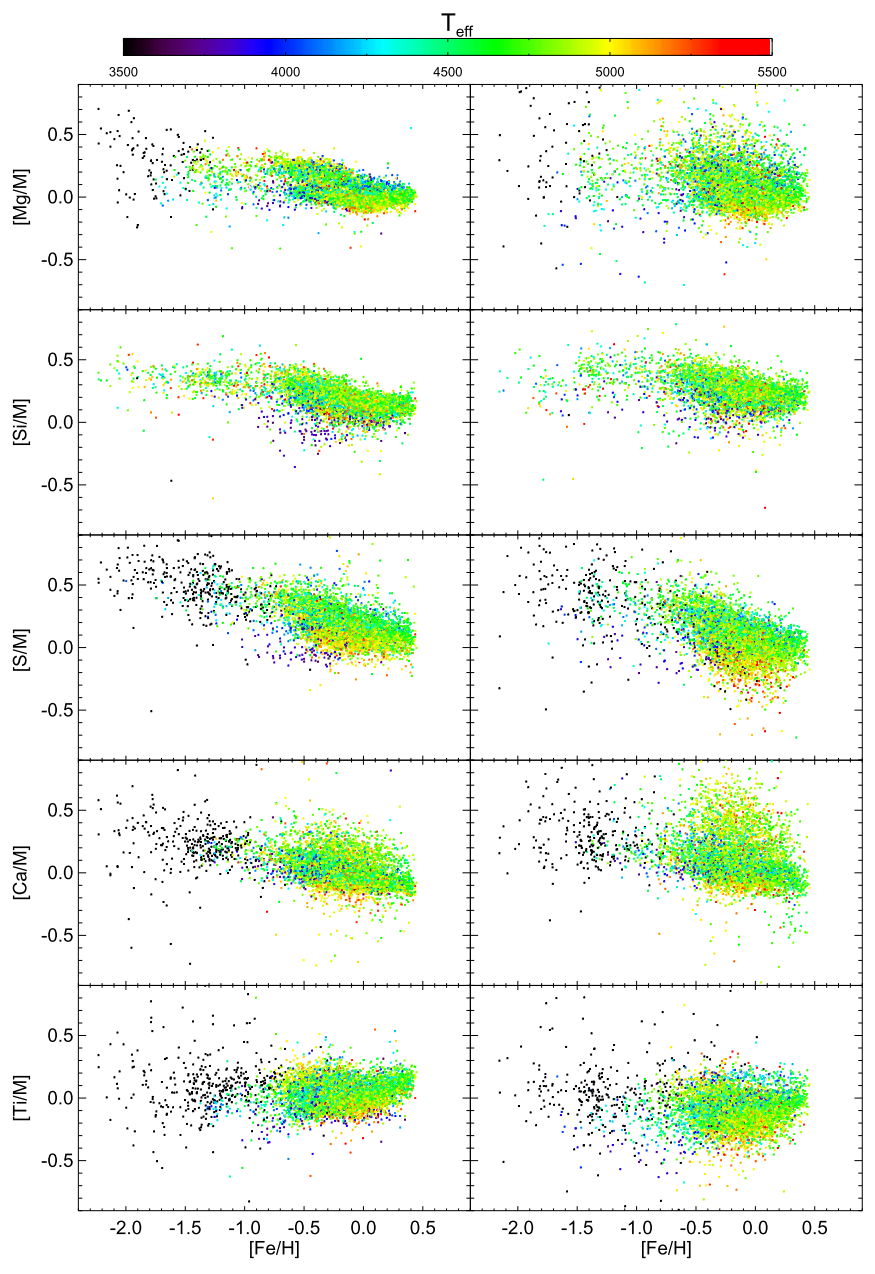

Figure 17. As Figure 16, but for $\alpha$-element abundances (Mg, Si, S, Ca, Ti).

There are also some tables that link these tables, e.g., apogeeStar to apogeeVisit.

\subsection{Science Archive Server (SAS)}

The data files created at multiple stages in the data reduction and processing pipeline are available on the SAS, which is accessible through the web at http://data.sdss3.org/sas/dr12.

\subsubsection{Summary FITS Tables}

Summary FITS tables are available that present compilations of data for all stars in the data release. These FITS tables are the original data source for the CAS database tables:

1. allStar-summary information on each individual star observed in SDSS-III/APOGEE, which includes mean barycentric radial velocity, ASPCAP parameters and abundances as derived from the summed spectra, and a compilation of ancillary targeting data (for DR12, allStarv603.fits);

2. allVisit - summary information on each individual visit of each star in SDSS-III/APOGEE, which includes the barycentric radial velocity of each visit, along with a compilation of ancillary targeting data (for DR12, allVisit-v603.fits). 


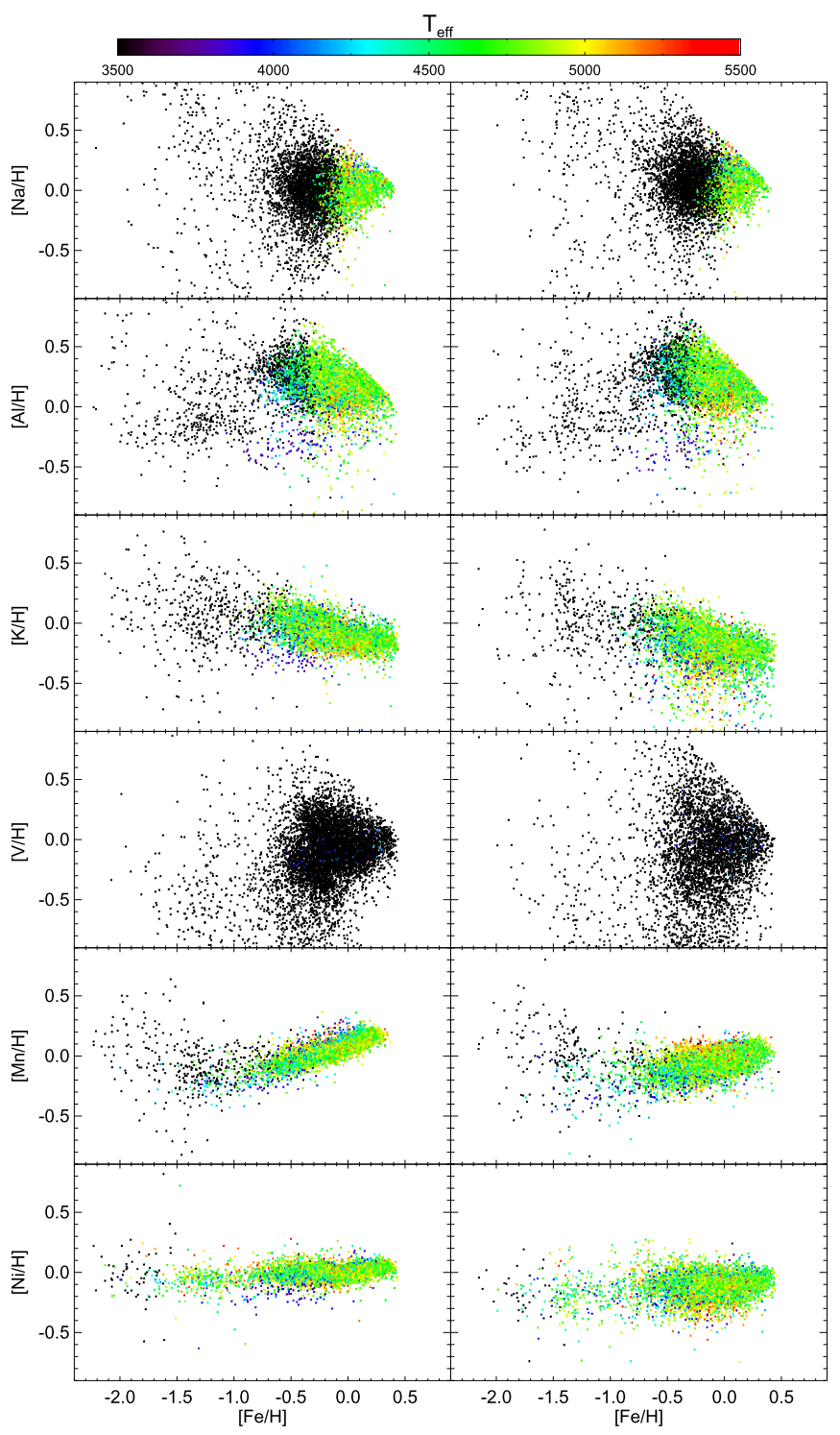

Figure 18. As Figure 16, but for $\mathrm{Na}, \mathrm{Al}, \mathrm{K}, \mathrm{V}, \mathrm{Mn}$, and Ni.

3. allPlates - summary information for all observed plates, include the location and plate design information (for DR12, allPlates-v603.fits).

Both allStar and allVisit files are FITS files with several header/data units (HDUs). The first HDU consists of the main data, with an entry for each star (allStar HDU1) or visit (allVisit HDU1). The objects are sorted by increasing right ascension. The second HDU in both files contains a 360 element integer array giving the HDU1 array index of the first object larger than each degree of right ascension, which may be useful for a rapid search through the table for an object of known R.A. The third HDU contains information about the order of array values in the PARAM (PARAM_SYMBOL) and ELEM (ELEM_SYMBOL and ELEM_VALUE) arrays in HDU1; these are constant for all objects and thus do not need to be repeated for each object.

The allStar file provides pointers into the allVisit file to identify the visits that went into the combined spectra.

There are also several summary targeting FITS tables:
1. apogeeDesign gives targeting information for each plate design

2. apogeePlate gives targeting information for each plate (design_id, location_id, hour angle, temperature, epoch)

3. apogeeField gives targeting information for each field (name, location_id, coordinates, and expected number of visits)

4. apogeeObject gives all of the ancillary catalog/targeting information for all stars in each field from which the observed targets were drawn.

Targeting terms are defined in Zasowski et al. (2013), particularly in the glossary of that paper.

\subsubsection{Spectral Data}

The spectral data files created at multiple stages in the data reduction and processing pipeline are also available on SAS. Some of the data that might be of the most interest include:

1. apVisit files-FITS image files with individual visit spectra of each star on each MJD in which it was observed;

2. apStar files-FITS image files with combined (across multiple visits) spectra of each star observed with APOGEE, along with uncertainty and mask arrays, as well as the individual visit spectra resampled to the apStar rest wavelength scale;

3. aspcapStar files-FITS image files for each star that give the continuum normalized spectra from which parameters and abundances are derived, the adopted uncertainties in the spectra, and the best-matching synthetic spectra, along with derived parameters and abundances in the image header;

4. apField files-FITS tables for each field that include information and the radial velocities for all stars in the field;

5. aspcapField files-FITS tables for each field that include the derived ASPCAP parameters and abundances for all stars in the field, along with the spectra and best-matching synthetic spectra.

These files are described in more detail in Section 8. Note that the information in the apField and aspcapField files is compiled including all fields in the allStar file discussed above.

In addition to these main products, the SAS contains all of the data from APOGEE, from the raw data cubes to the final processed spectra and derived parameters, including all of the intermediate data products.

Table 9 provides a rough guide to the overall data structure in the SAS. All of these data products are documented through the SDSS datamodel, available at http://data.sdss3.org/ datamodel.

\subsection{Web Interface to the SAS}

A web application provides an interface to the SAS through a front-end database called the SAS database (SASDB). This provides a tool by which individual spectra can be interactively inspected without having to download the data, and also provides an interface by which you can search for data to download either for individual objects, or for a bulk search of multiple objects. This can be found at http://data.sdss3.org. 
Table 9

SAS Directory Structure

\begin{tabular}{|c|c|}
\hline Directory & Contents \\
\hline APRED_VERS & top level directory for a given visit reduction version \\
\hline APRED_VERS/red/MJD & $\begin{array}{l}\text { reduced data for individual exposures, } \\
\text { ap2D and ap1D }\end{array}$ \\
\hline APRED_VERS/apo25m/PLATE/MJD & reduced visit data for $2.5 \mathrm{~m}$ data: apVisit files \\
\hline APRED_VERS/apo1m/PLATE/MJD & reduced visit data for $1 \mathrm{~m}$ data: apVisit files \\
\hline APRED_VERS/APSTAR_VERS & top level for a given star combination version \\
\hline APRED_VERS/APSTAR_VERS/apo25m/LOCATION_ID & combined, resampled spectra: apStar files for $2.5 \mathrm{~m}$ data \\
\hline APRED_VERS/APSTAR_VERS/apo1m/PROGRAM_ID & combined, resampled spectra: apStar files for $1 \mathrm{~m}$ data \\
\hline APRED_VERS/APSTAR_VERS/ASPCAP_VERS & $\begin{array}{l}\text { top level directory for a given ASPCAP } \\
\text { version/configuration }\end{array}$ \\
\hline APRED_VERS/APSTAR_VERS/ASPCAP_VERS/location_id & FERRE files for a given location \\
\hline APRED_VERS/APSTAR_VERS/ASPCAP_VERS/RESULTS_VERS & $\begin{array}{l}\text { top level directory for a given RESULTS (calibration) } \\
\text { version: allStar and allVisit summary tables }\end{array}$ \\
\hline APRED_VERS/APSTAR_VERS/ASPCAP_VERS/RESULTS_VERS/location_id & $\begin{array}{l}\text { ASPCAP results in FITS files for given field: } \\
\text { aspcapStar and aspcapField files }\end{array}$ \\
\hline
\end{tabular}

\section{USING APOGEE DATA}

\subsection{Bitmasks, Data Quality, and Target Classes}

SDSS uses bitmasks to flag stars with quality issues that may affect the accuracy of the derived parameters and abundances, as well as to provide information on targeting. A bitmask is an integer in which individual bits (corresponding to powers of two in a decimal representation) can be set to indicate a variety of conditions. Users are advised to routinely check the contents of these.

\subsubsection{Targeting Bitmasks}

There are different target classes for objects that appear in APOGEE data releases. While the bulk of the targets were chosen for the "main" survey (i.e., stars selected with a simple color selection), there are a number of other target classes, as described in detail in Zasowski et al. (2013). These include special targets such as the cluster targets used for the calibration, targets for ancillary science programs, and observations obtained with the NMSU $1 \mathrm{~m}$ feed to the APOGEE spectrograph. The targeting class for each target is documented in a pair of targeting bitmasks, APOGEE_TARGET1 and APOGEE_TARGET2, where the bit definitions are defined in Zasowski et al. (2013) and documented at http://www.sdss.org/dr12/algorithms/ bitmasks/\#APOGEE_TARGET1 and http://www.sdss.org/ dr12/algorithms/bitmasks\#APOGEE_TARGET2. We provide character "translations" of the targeting bitmasks in the APOGEE_TARGFLAGS column in summary data files.

Note that the main survey targets are not defined by a single bit in the targeting bitmasks; instead, they can be identified as targets that have one of the APOGEE_SHORT, APOGEE_INTERMEDIATE, or APOGEE_LONG bits set. For convenience, we have included a separate bitmask, EXTRATARG, in the summary files and in the CAS tables. This bitmask has a value of zero for all main survey targets, but has different bits set if the target is either a star selected for science outside of the main survey (bit 0 ), commissioning data (bit 1), a telluric star (bit 2), or a star observed with the NMSU $1 \mathrm{~m}$ (bit 3), see http://www. sdss.org/dr12/algorithms/bitmasks\#APOGEE_EXTRATARG.

There are some objects that may appear more than once in the summary files or the CAS tables. Many of these are stars that were observed during the commissioning period. These observations are kept separate because the quality of the data is poorer (worse LSF). Since these data are not homogeneous with the main survey data, they are not analyzed with ASPCAP; they are included because they still provide useful radial velocity information and may reveal spectral variations over time (e.g., double-line spectroscopic binaries or emission line stars). Apart from this, there are $~ 1600$ stars that appear more than once with survey data. This occurs because these objects were targeted in different, but overlapping fields. In some cases, this was intentional (in particular, for some of the ancillary science programs), but for others the targets were inadvertently selected independently in two different overlapping fields. For all duplicate targets, we choose a "primary" observation as the observation with the highest $\mathrm{S} / \mathrm{N}$, and all other observations of this target have a bit (bit 4) set in the EXTRATARG bitmask.

The most homogeneous sample are those stars with EXTRATARG $=0$. This will give only main survey targets, excluding all special targets, commissioning data, $1 \mathrm{~m}$ objects, telluric stars, and duplicate targets.

\subsubsection{STARFLAG Bitmask}

Each visit spectrum has an associated STARFLAG bitmask, which flags a number of conditions as given in Table 10 and at http://www.sdss.org/dr12/algorithms/ bitmasks/\#APOGEE_STARFLAG. If BAD_PIXELS, VERY_BRIGHT_NEIGHBOR, or LOW_SNR is set for a visit spectrum, that spectrum is deemed bad and not used to obtain the final combined spectrum. The PERSIST_HIGH, PERSIST_MED, and PERSIST_LOW bits flag stars that may be significantly affected by the persistence on the blue detector; they are set if $20 \%$ or more pixels are in the persisence region, with the three different levels representing different amplitude of the persistence affect (note that PERSIST_LOW is still significant persistence: stars outside the persistence region altogether do not have any of the these bits set). The most conservative sample with regard to persistence would not have any of these three bits set.

The spectra that are combined from the individual visit spectra have STARFLAG set to the logical OR of the STARFLAG values of the individual visits. In addition, there is another ANDFLAG that is set to the logical AND of the flags from the individual visits. 
Table 10

STARFLAG Bitmask

\begin{tabular}{|c|c|c|}
\hline Condition & Bit & Description \\
\hline BAD_PIXELS & 0 & Spectrum has many bad pixels $(>40 \%)$ : BAD \\
\hline COMMISSIONING & 1 & Commissioning data (MJD < 55761), non-standard configuration, poor LSF: WARN \\
\hline BRIGHT_NEIGHBOR & 2 & Spectrum has neighbor on detector more than 10 times brighter: WARN \\
\hline VERY_BRIGHT_NEIGHBOR & 3 & Spectrum has neighbor on detector more than 100 times brighter: BAD \\
\hline \multirow[t]{2}{*}{ LOW_SNR } & 4 & Spectrum has low $\mathrm{S} / \mathrm{N}(\mathrm{S} / \mathrm{N}<5)$ : BAD \\
\hline & $5-8$ & Currently unused \\
\hline PERSIST_HIGH & 9 & Spectrum has significant number $(>20 \%)$ of pixels in high persistence region: WARN \\
\hline PERSIST_MED & 10 & Spectrum has significant number $(>20 \%)$ of pixels in medium persistence region: WARN \\
\hline PERSIST_LOW & 11 & Spectrum has significant number $(>20 \%)$ of pixels in low persistence region: WARN \\
\hline PERSIST_JUMP_POS & 12 & Spectrum shows obvious positive jump in blue chip: WARN \\
\hline \multirow[t]{2}{*}{ PERSIST_JUMP_NEG } & 13 & Spectrum shows obvious negative jump in blue chip: WARN \\
\hline & $14-15$ & Currently unused \\
\hline SUSPECT_RV_COMBINATION & 16 & $\begin{array}{l}\text { WARNING: RVs from synthetic template differ significantly } \\
\text { from those from combined template }\end{array}$ \\
\hline \multirow[t]{2}{*}{ SUSPECT_BROAD_LINES } & 17 & $\begin{array}{l}\text { WARNING: cross-correlation peak with template significantly } \\
\text { broader than autocorrelation of template }\end{array}$ \\
\hline & $18-31$ & Currently unused \\
\hline
\end{tabular}

Table 11

ASPCAPFLAG Bitmask

\begin{tabular}{|c|c|c|}
\hline Condition & Bit & Description \\
\hline TEFF_WARN & 0 & WARNING on effective temperature (see PARAMFLAG[0] for details) \\
\hline LOGG_WARN & 1 & WARNING on $\log g$ (see PARAMFLAG[1] for details) \\
\hline VMICRO_WARN & 2 & WARNING on vmicro (see PARAMFLAG[2] for details) \\
\hline M_H_WARN & 3 & WARNING on [M/H] (see PARAMFLAG[3] for details) \\
\hline ALPHA_M_WARN & 4 & WARNING on $[\alpha / \mathrm{M}]$ (see PARAMFLAG[4] for details) \\
\hline C_M_WARN & 5 & WARNING on [C/M] (see PARAMFLAG[5] for details) \\
\hline N_M_WARN & 6 & WARNING on [N/M] (see PARAMFLAG[6] for details) \\
\hline STAR_WARN & 7 & $\begin{array}{l}\text { WARNING overall for star: set if any of TEFF, } \\
\text { LOGG, CHI2, COLORTE, ROTATION, SN warn are set }\end{array}$ \\
\hline CHI2_WARN & 8 & high $\chi^{2}\left(>2^{*}\right.$ median at ASPCAP temperature) (WARN) \\
\hline COLORTE_WARN & 9 & $\begin{array}{l}\text { effective temperature more than } 1000 \mathrm{~K} \text { from photometric } \\
\text { temperature for dereddened color (WARN) }\end{array}$ \\
\hline ROTATION_WARN & 10 & $\begin{array}{l}\text { Spectrum has broad lines, with possible bad effects: ratio of FWHM of cross-correlation } \\
\text { of spectrum with best RV template to FWHM of auto-correlation of template }>1.5 \text { (WARN) }\end{array}$ \\
\hline \multirow[t]{2}{*}{ SN_WARN } & 11 & $\mathrm{~S} / \mathrm{N}<70(\mathrm{WARN})$ \\
\hline & $12-15$ & Not currently used \\
\hline TEFF_BAD & 16 & BAD effective temperature (see PARAMFLAG[0] for details) \\
\hline LOGG_BAD & 17 & BAD log g (see PARAMFLAG[1] for details) \\
\hline VMICRO_BAD & 18 & BAD vmicro (see PARAMFLAG[2] for details) \\
\hline M_H_BAD & 19 & BAD $[\mathrm{M} / \mathrm{H}]$ (see PARAMFLAG[3] for details) \\
\hline ALPHA_M_BAD & 20 & BAD $[\alpha / \mathrm{M}]$ (see PARAMFLAG[4] for details) \\
\hline C_M_BAD & 21 & BAD [C/M] (see PARAMFLAG[5] for details) \\
\hline N_M_BAD & 22 & BAD [N/M] (see PARAMFLAG[6] for details) \\
\hline STAR_BAD & 23 & $\begin{array}{l}\text { BAD overall for star: set if any of TEFF, LOGG, CHI2, COLORTE, ROTATION, } \\
\text { SN error are set, or any parameter is near grid edge (GRIDEDGE_BAD is set in any PARAMFLAG) }\end{array}$ \\
\hline CHI2_BAD & 24 & high $\chi^{2}\left(>5^{*}\right.$ median at ASPCAP temperature) (BAD) \\
\hline COLORTE_BAD & 25 & effective temperature more than $2000 \mathrm{~K}$ from photometric temperature for dereddened color (BAD) \\
\hline ROTATION_BAD & 26 & $\begin{array}{l}\text { Spectrum has broad lines, with possible bad effects: ratio of FWHM of cross-correlation } \\
\text { of spectrum with best RV template to FWHM to auto-correlation of template }>2 \text { (BAD) }\end{array}$ \\
\hline \multirow[t]{2}{*}{ SN_BAD } & 27 & $\mathrm{~S} / \mathrm{N}<50(\mathrm{BAD})$ \\
\hline & $28-30$ & Not currently used \\
\hline NO_ASPCAP_RESULT & 31 & True for all commissioning data, and for spectra that are marked as bad \\
\hline
\end{tabular}

\subsubsection{ASPCAP Bitmasks}

Several bitmasks are used in the ASPCAP processing. There is an overall ASPCAPFLAG, which flags various conditions as listed in Table 11. In particular, bits are set if any of the parameters are near the spectral library grid edges, if the ASPCAP temperature differs significantly from that expected from the observed colors, if the spectrum shows signs of significant stellar rotation, or if the spectrum does not meet the survey requirement for $\mathrm{S} / \mathrm{N}$.

Of particular note is the STAR_BAD bit, which is set if any of other bits (TEFF_BAD, LOGG_BAD, CHI2_BAD, COLORTE_BAD, ROTATION_BAD, SN_BAD) to denote 
Table 12

PARAMFLAG Bitmask

\begin{tabular}{lcl}
\hline \hline Condition & Bit & Description \\
\hline GRIDEDGE_BAD & 0 & $\begin{array}{l}\text { Parameter within 1/8 grid spacing of } \\
\text { grid edge } \\
\text { Parameter outside valid range of calibra- } \\
\text { tion determination } \\
\text { Other error condition }\end{array}$ \\
OTHER_BAD & 2 & $\begin{array}{l}\text { Not currently used } \\
\text { Parameter within 1/2 grid spacing of } \\
\text { grid edge }\end{array}$ \\
GRIDEDGE_WARN & $3-7$ & $\begin{array}{l}\text { Parameter in possibly unreliable range of } \\
\text { calibration determination }\end{array}$ \\
CALRANGE_WARN & 9 & $\begin{array}{l}\text { Other warning condition } \\
\text { Not currently used }\end{array}$ \\
OTHER_WARN & $10-15$ & Parameter set at fixed value, not fit \\
PARAM_FIXED & 16 & Not currently used
\end{tabular}

that the ASPCAP results are very likely not to be reliable. Stars with this bit set should not be used for parameter/abundance analysis. Note that some bits in ASPCAPFLAG are informational/warning only, so restricting a sample to ASPCAPFLAG $=0$ is not an appropriate filter to get good data.

In addition, there are PARAMFLAG and ELEMFLAG arrays for each of the stellar parameters and individual element abundances that provide additional information about why a given parameter may be denoted BAD in ASPCAPFLAG or that flag potential issues with the abundance determination for individual elements. Table 12 gives the definition of the PARAMFLAG bits.

\subsection{Radial Velocities}

Several measurements of radial velocities are provided, as discussed in the following subsections.

\subsubsection{Systemic Radial Velocities}

The average systemic velocity for each APOGEE star is given in VHELIO_AVG; estimated uncertainties are given in VERR (weighted error), and VERR_MED (median error of individual visit RVs). The scatter obtained from multiple visits (with number of visits given in NVISITS) is given in VSCATTER. Note that objects with VSCATTER $>0.5 \mathrm{~km} \mathrm{~s}^{-1}$ are potentially $\mathrm{RV}$-variable objects, based on the typical RV accuracy (see Nidever et al. 2015). These velocities were determined by deriving relative visit RVs by iterative cross-correlation of each visit against the combined spectrum for the object, with an absolute scale established after convergence by cross-correlation of combined spectra with the best-matching synthetic spectrum in a RV template grid. This has the advantage of having no template mismatch for the relative RVs (although a disadvantage is that the combined template is not noiseless). The parameters of the best-matching grid point are given in RV_TEFF, RV_LOGG, and RV_FEH, but these are not to be confused with the much better determined ASPCAP parameters; no interpolation is performed in the RV grid.

An alternative systemic velocity is given in SYNTHVHELIO_AVG, with uncertainties in SYNTHVERR and SYNTHVERR_MED and scatter in SYNTHVSCATTER. These are determined by cross-correlation of each visit with the RV template grid, and then subsequent combination.
The scatter between the two different systemic RV measurements is given in SYNTHSCATTER. Larger values here suggest possible issues with the derived RVs. For such objects, the SUSPECT_RV_COMBINATION bit in the STARFLAG bitmask is set.

\subsubsection{Individual Visit Radial Velocities}

The individual visit radial velocities are stored in the visit tables (allVisit FITS table in the SAS, and the allVisit table in the CAS). The visit RV information derived from crosscorrelating each visit against the combined spectra are stored with labels VREL (the measured RV, uncorrected for Earth motion), VRELERR (uncertainty in VERR), and VHELIO (measured RV corrected for barycentric motion; we note the slight misnomer in the VHELIO label since these are strictly barycentric, not heliocentric velocities). The visit RVs derived from cross-correlating each visit against the best-matching template are stored in SYNTHVREL, SYNTHVRELERR, and SYNTHVHELIO.

An initial estimate of the radial velocity for each for each visit is made by cross-correlation of the individual visit spectra with the same coarse grid of synthetic templates mentioned above. These are stored in the visit tables as ESTVREL, ESTVRELERR, and ESTVHELIO.

\subsection{Stellar Parameters}

As described previously, each APOGEE spectrum is matched to a spectral grid covering several dimensions $-T_{\text {eff }}$, $\log g, v_{\text {micro }},[\mathrm{M} / \mathrm{H}],[\mathrm{C} / \mathrm{M}],[\mathrm{N} / \mathrm{M}],[\alpha / \mathrm{M}]$ — to derive a set of stellar parameters that are subsequently used for the individual element abundance fits.

Calibrations to $T_{\text {eff }}, \log g,[\mathrm{M} / \mathrm{H}]$, and $[\alpha / \mathrm{M}]$, as described above, are applied to the raw FERRE output; for $[\mathrm{M} / \mathrm{H}]$, both internal (temperature) and external calibration is applied. These quantities are stored in the PARAM array in the allStar file (HDU1); the order of the parameters in the array is given in the PARAM_SYMBOL array in HDU3 of the allStar file. In the CAS, the parameters are given in individual columns PARAM_TEFF, PARAM_LOGG, PARAM_LOGVMICRO, PARAM_M_H, PARAM_C_M, PARAM_N_M, and PARAM_ALPHA_M in the aspcapStar table.

The calibrated effective temperatures and surface gravities are duplicated in columns TEFF and LOGG in both the allStar file and CAS tables. These are the recommended values for these quantities. The allStar file also duplicates the parameterlevel $[\mathrm{M} / \mathrm{H}]$ and $[\alpha / \mathrm{M}]$ in PARAM_M_H and PARAM_ALPHA_M.

The raw, uncalibrated FERRE output is stored in an FPARAM array in the allStar file, and in the CAS in columns FPARAM_TEFF, FPARAM_LOGG, FPARAM_M_H, FPARAM_C_M, FPARAM_N_M, and FPARAM_ALPHA_M in the aspcapStar table.

There is a distinction between the parameter-level $[\mathrm{M} /$ $\mathrm{H}]$ and $[\alpha / \mathrm{M}]$ values and the individual elemental abundances discussed below. The parameter-level quantities are fits to the entire spectrum, and might weight different elements differently over a range of temperatures. However, they might also carry higher accuracy as averages over multiple elements and more wavelengths. Furthermore, the parameter-level $[\mathrm{M} / \mathrm{H}]$ is the only abundance that is externally calibrated, based on the literature cluster metallicities discussed previously. 
Stars with unreliable parameters are flagged by having the STAR_BAD bit in the ASPCAPFLAG bitmask set. If this bit is set, then other bits in ASPCAPFLAG can be used to determine the reason for the measurements being flagged as unreliable. The STAR_WARN bit can be consulted to determine if derived parameters may be suspect based on less stringent criteria.

As discussed previously, while the ASPCAP results can easily discriminate dwarfs from giants, the results for dwarfs are significantly less secure and lack an extensive calibration sample. As a result, no calibratrated parameters are provided for dwarfs.

Based on the quality of the fits and external comparisons, we also note that ASPCAP results at cooler temperatures, $T_{\text {eff }}<$ $4000 \mathrm{~K}$ may be more uncertain.

\subsection{Stellar Abundances}

For the 15 individual elemental abundances, the internal calibration (Section 5.4.1) of abundance with temperature is applied, and the results are stored in the ELEM array in the allStar file (HDU1); the order of the elements in the array is by atomic number and is given in the ELEM_SYMBOL array in HDU3 of the allStar file. Since carbon, nitrogen, and the $\alpha$ elements are fit by varying the $[\mathrm{C} / \mathrm{M}],[\mathrm{N} / \mathrm{M}]$, and $[\alpha / \mathrm{M}]$ dimensions in the spectral library, respectively, the abundances from FERRE for these elements are relative to the overall scaled-solar metal content, $[\mathrm{M} / \mathrm{H}]$, while the abundances for other elements are relative to hydrogen since they are fit by varying $[\mathrm{M} / \mathrm{H}]$. These differences in the array contents are documented in the ELEM_VALUE array of HDU3, which explicitly gives what values are presented in the arrays.

For convenience, we convert all calibrated abundances into $[\mathrm{X} / \mathrm{H}]$ values (by adding $[\mathrm{M} / \mathrm{H}]$ to the carbon, nitrogen and $\alpha$ element abundances) and store these in named quantities: $\mathrm{C} \_\mathrm{H}$, N_H, O_H, NA_H, MG_H, AL_H, SI_H, S_H, K_H, CA_H, TI_H, V_H, MN_H, FE_H, NI_H in the allStar file and also as individual columns in the aspcapStar table in the CAS. As discussed in Section 5.4.3, these abundances do not have any external calibration applied to them.

The uncalibrated abundances are stored in an FELEM array in the allStar file, and in individual columns FELEM_C_M, FELEM_N_M, FELEM_O_M, FELEM_NA_H, FELEM_MG_M, FELEM_AL_H, FELEM_SI_M, FELEM_S_M, FELEM_K_H, FELEM_CA_M, FELEM_TI_M, FELEM_V_H, FELEM_MN_H, FELEM_FE_H, FELEM_NI_H in the CAS table. Note that these are not all converted into $[\mathrm{X} / \mathrm{H}]$; as raw values, they preserve the way in which they were derived.

The ASPCAPFLAG should be consulted to confirm that there are no significant issues with the stellar parameters. In addition, before using the abundance of any particular element, it is important to confirm that the derived value is not near an abundance grid edge. This is achieved by consulting the appropriate ELEMFLAG bitmask, looking to see if the GRIDEDGE_BAD bit (value within $1 / 8$ of a grid spacing of edge) or the GRIDEDGE_WARN bit (value within $1 / 2$ of a grid spacing of edge) is set. This is especially critical when looking at abundance ratios, where the value of the ratio may not immediately indicate a problem with one or both of the abundances.

Similarly to the stellar parameters, abundances for dwarfs are more uncertain, and calibrated abundances for dwarfs are not provided.
The calibrated giant abundances have an effective temperature correction applied, as described in Section 5.4. Since this metallicity-independent linear correction is perhaps unlikely to represent the full behavior of the abundances, a sample that covers a wide temperature range may have larger possibility for temperature-dependent systematics than one that is restricted to a smaller temperature range. In addition, the calibration sample only extends down to $T_{\text {eff }} \sim 3800 \mathrm{~K}$, so the behavior at cooler temperatures is less well known; the current results apply the offset at $3800 \mathrm{~K}$ for all cooler stars, but set the CALRANGE_WARN bit in the ELEMFLAG bitmask.

Results for some elements may be less reliable than for others. In particular, the titanium abundances show large scatter compared with previous measurements for a calibration subsample, and do not show the expected behavior with overall metallicity. Other elements like sodium and vanadium have relatively few and weak features, and thus have larger uncertainties.

In addition, as noted in Section 5.7, individual element abundances for objects whose spectra fell in the the persistence region of the blue detector may be more uncertain. These objects can be identified by those having one of the PERSIST_HIGH, PERSIST_MED, or PERSIST_LOW bits set in the ANDFLAG bitmask (for objects falling in persistence region in all visits), or those with one of the persistence bits set in the STARFLAG bitmask (for objects falling in persistence region in any of the visits).

The empirical uncertainty estimates derived from simple fits to the observed scatter of abundances in clusters as a function of temperature, metallicity and signal-to-noise (see Section 5.4.1) are stored in the ELEM_ERR array (and correspondingly named columns X_H_ERR in the CAS). Note that the scatter used for these estimates is the scatter in the raw FERRE abundance measurements, i.e., in $[\mathrm{X} / \mathrm{M}]$ for $\mathrm{C}, \mathrm{N}$ and the $\alpha$ elements, but in $[\mathrm{X} / \mathrm{H}]$ for $\mathrm{Na}, \mathrm{Al}, \mathrm{K}, \mathrm{V}, \mathrm{Mn}, \mathrm{Fe}$, and $\mathrm{Ni}$. The FELEM_ERR array (and corresponding column names in the CAS) gives the raw FERRE uncertainties, but as discussed previously, these seem to significantly underestimate the true uncertatinties.

For uncertainties in abundance ratios, it is not necessarily appropriate to add the uncertainties of the individual abundances in quadrature, as the scatter may not all result from random errors. If there are systematic errors contributing to the scatter, it is possible that different elements will be offset in the same way, such that abundance ratios could be more robust. Further analysis on this issue is planned for the future.

\subsection{Selection Effects}

As with all surveys, there are selection effects in the APOGEE catalog. Several effects to consider are:

1. Main sample selection: only a fraction of the stars in each field are selected for observation, with a well-defined distribution in magnitude and color-see Zasowski et al. (2013) for details.

2. Ancillary and calibration targets: in addition to the main survey targets, there are calibration and ancillary program targets that are not selected by the usual algorithm. While the non-main survey targets can be recognized from the bitmasks (e.g., EXTRATARG $=0$ ), there is a subtle effect that any targets that are part of an ancillary program are rejected from being selected as a part of the main 
sample, so it is possible that some object types are actually underrepresented in the main sample.

3. Abundance biases at warmer temperatures: the sample selection generally uses a (dereddened) blue color cut of $(J-K)_{0}>0.5$. While this was designed to include metal-poor luminous giants, it does bias against lower metallicity stars on the lower giant branch. Furthermore, the abundance measurements may become less certain at warmer temperatures because features are weaker.

4. Abundance biases at cooler temperatures: the current spectral grids are limited to $T_{\text {eff }}>3500 \mathrm{~K}$ and $\log g>0$, so no cooler and lower surface gravity stars appear with good ASPCAP parameters. This biases against the most metal-rich stars, in particular, for the most luminous giants as well as bright AGB stars. Furthermore, the abundance measurements may become less certain at cooler $\left(T_{\text {eff }}<4000 \mathrm{~K}\right)$ temperatures because of the complexity of the spectra and current limitations in our ability to model it well.

\subsection{Caveats}

There are issues that are discovered in public data releases after the point when things can be modified. The online SDSS / APOGEE documentation attempts to track these at http:// www.sdss3.org/dr12/irspec/caveats. At the time of the data release, known caveats include:

1. Uncertainties given as $[\mathrm{X} / \mathrm{H}]$ are actually computed from $[\mathrm{X} / \mathrm{M}]$ scatter for $\mathrm{C}, \mathrm{N}$ and $\alpha$ elements.

2. Character translations for target flags (in APOGEE_TARGFLAGS) are missing in the apVisit and allVisit files for four ancillary programs (APOGEE_RV_MONITOR_IC348, APOGEE_RV_MONITOR_KEPLER, APOGEE_GES_CALIBRATE, APOGEE_BULGE_ RV_VERIFY); they are correct in the apStar and allStar file.

3. Some targets observed in multiple fields and appear as duplicates (as flagged with the EXTRATARG flag discussed previously).

4. Some targets may have been selected independently for different programs within different visits to the same field. As a result, there are stars constructed from multiple visits for which a target flag bit may be set in the combined spectra, but not in all of the visit spectra that were used to construct it.

5. The APOGEE_ANCILLARY bit is not set for APOGEE_KEPLER_SEISMO and APOGEE_KEPLER_HOST targets.

6. For a few objects, slightly incorrect object/star names were used during the reduction.

\section{USING APOGEE SPECTRA}

\subsection{Raw Data}

Raw data files are saved as $a p R$ - $a b c]-I D 8 . a p z$ frames, where $[a b c]$ refers to each of the three chips, and the ID8 is an eight digit number that uniquely identifies each APOGEE exposure. The first four digits give the number of days since 2010 December 31, i.e., MJD-55562. The last four digits are a running number of the frames taken during that day.
Table 13

Contents of apVisit Files

\begin{tabular}{ll}
\hline \hline HDU & Contents \\
\hline 0 & main header with target information, starflag \\
1 & flux \\
2 & uncertainty \\
3 & pixel bitmask \\
4 & wavelength \\
5 & sky \\
6 & sky uncertainty \\
7 & telluric correction spectrum \\
8 & telluric correction uncertainty \\
9 & wavelength coefficients \\
10 & LSF coefficients \\
\hline
\end{tabular}

Data from the three detectors are stored in separate FITS files, with the longest wavelengths at the lowest columns in chip $a$ and the shortest wavelengths at the highest columns in chip $c$. Each file is compressed using the FPACK algorithm (Seaman et al. 2010); the resulting images are given as an initial image plus a set of differences between adjacent up-theramp reads. We highlight the fact that some reconstruction is necessary to give the raw data cubes by giving the $a p R$ files a .apz extension name (rather than .fits), even though they are valid FITS files. See Nidever et al. (2015) for more details.

\subsection{Visit Spectra}

Individual visit spectra of each star on each MJD in which it was observed are saved in apVisit-VERS-PLATE-MJD-FIBER. fits files. VERS represents the data release version of the reduction routines, which is $\mathrm{r} 5$ for the DR12 release. PLATE is the SDSS plate identification that corresponds to a physical set of holes into which fibers are plugged. MJD represents the modified Julian date of the observations, and FIBER represents which fiber was used to obtain the spectrum. The FIBER number is defined such that the spectrum at the highest row numbers has FIBER $=1$, while those at the lowest row numbers have FIBER $=300$ (see S. R. Majewski et al. 2015, in preparation). The association of the fiber number with an object name is provided by a "plugmap" file; this association is used to populate header information with an object name and coordinates.

The apVisit spectra combine the multiple dithered exposures taken on a given night. They are on the native APOGEE pixel scale, but with half-pixel sampling made possible by the dithered exposures. Multiple extensions give uncertainty in the spectra, a bitmask flag for individual pixel status (http://www. sdss.org/dr12/algorithms/bitmasks/\#APOGEE_PIXMASK), a wavelength array, the sky and telluric spectra used to correct the object spectrum, and some other ancillary information. The content of the files is summarized in Table 13.

There will be some bad pixels in any given spectrum that result from bad pixels on the APOGEE detectors. Such pixels are flagged in the pixel bitmask that is stored in HDU3 of the apVisit files, which encodes the reason why any given pixel is flagged as unreliable. The bitmask definitions for these are given at http://www.sdss.org/dr12/algorithms/bitmasks/ \#APOGEE_PIXMASK. The bitmask also encodes locations where data may be more suspect, e.g., pixels in the strong persistence regions of the detectors, and/or pixels in the 
Table 14

Contents of apStar Files

\begin{tabular}{ll}
\hline \hline HDU & Contents \\
\hline 0 & main header with target, RV, version information \\
1 & resampled spectra \\
2 & uncertainties in spectra \\
3 & pixel mask for spectra \\
4 & sky spectrum \\
5 & sky uncertainty \\
6 & telluric spectrum \\
7 & telluric uncertainty \\
8 & LSF coefficients \\
9 & RV information, including template \\
& cross-correlation and autocorrelation \\
\hline
\end{tabular}

location of the Littrow ghost (see J. Wilson et al. 2015, in preparation).

While correction is made for telluric absorption and for sky emission, these corrections (especially for sky emission) are generally imperfect. As a result, users can expect to see some residual sky emission in the visit spectra. Locations of strong sky emission and telluric absorption are flagged in the pixel bitmask.

\subsection{Combined Spectra}

FITS image files with combined (across multiple visits) spectra of each star observed with APOGEE are saved in apStar-VERS-APOGEE_ID.fits files. Here, VERS represents the reduction version (again, $\mathrm{r} 5$ for the DR12 release) and APOGEE_ID represents an object identifier, which is usually the 2MASS object name with a " $2 \mathrm{M}$ " prefix; for the few objects that are not in the 2MASS catalogs, a similar style APOGEE_ID was created from the coordinates, and a "AP" prefix was prepended.

The apStar files represent the main survey output spectra. These FITS files have multiple HDUs: they include the combined spectra and individual resampled visit spectra, uncertainty arrays corresponding to these, a pixel mask array that flags pixels with various conditions, a wavelength array, and other information, as summarized in Table 14.

The combined spectrum is included along with the individual visit spectra shifted to rest wavelength in HDU1. This is a 2D image with wavelength running along the column axis, and with NVISITS +2 rows that have two versions of the combined spectra in the first two rows, followed by NVISITS rows of resampled individual-visit spectra. The two combinations are generally very similar; they differ in the weighting scheme used to do the combination (see Nidever et al. 2015). The ASPCAP analysis of these spectra uses the first version of the combined spectra. Bad pixels in any of the individual visit spectra are discarded during the combination.

All spectra are put on a common wavelength scale with a fixed dispersion in $\log \lambda$ : apStar spectra all have 8575 pixels with $\log \lambda_{i}=4.179+6 . \times 10^{-6} i$, where $i$ is the pixel number (with the first pixel having $i=0$ ). This corresponds to a pixel sampling of $4.145 \mathrm{~km} \mathrm{~s}^{-1}$ and provides roughly 3 pixels per resolution element.

Because of the resampling, the uncertainties are correlated between adjacent pixels; the covariance information is not currently being tracked. If data with non-correlated errors are required, users are advised to use the apVisit files, but these
Table 15

Contents of aspcapStar Files

\begin{tabular}{ll}
\hline \hline HDU & Contents \\
\hline 0 & Normalized APOGEE spectrum \\
1 & Uncertainties in normalized spectrum \\
2 & Best library match as derived by ASPCAP/FERRE \\
3 & FITS table with parameters of best fit spectrum \\
\hline
\end{tabular}

Table 16

Contents of aspcapField Files

\begin{tabular}{ll}
\hline \hline HDU & Contents \\
\hline 0 & $\begin{array}{l}\text { FITS table with parameters and abundances for all } \\
\text { objects in field }\end{array}$ \\
& FITS table with normalized spectra, uncertainty array, \\
& and best-fit spectra \\
& FITS table giving the order of the parameters and \\
& abundances in the arrays of HDU0
\end{tabular}

have non-constant dispersion and, even here, there will be small covariances in the dither-combined images because the dithers are not perfect.

Because spectra are obtained with visits taken over a period of time, telluric absorption and sky emission features generally do not fall at the same rest wavelength in the stellar spectra because of the changing barycentric correction. This means that systematic errors in the correction of these features are generally reduced compared to the individual visit spectra, but are spread over a larger region of the stellar spectra. The pixel bitmask in HDU3 of the apStar files track pixels that were affected in the individual visit spectra.

\subsection{ASPCAP Spectra and Best Fits}

The aspcapStar files are FITS image files that give the continuum normalized spectra from which parameters and abundances are derived. These are given on the apStar wavelength scale, but only include the specific wavelength ranges that are matched to the synthetic grids (see A. E. García Pérez et al. 2015, in preparation for exact wavelength range). Multiple extensions give the best-matching synthetic spectra from which the stellar parameters are adopted (see Table 15).

\subsection{ASPCAP Field Results}

The aspcapField files are FITS tables for each field that include the derived ASPCAP parameters and abundances for all stars in the field. A second extension includes a FITS table with all of the normalized spectra and the best-matching synthetic spectra for all stars in the field. Because these files include spectra for hundreds of stars, they are moderately large (see Table 16).

\section{CONCLUSION}

We have described the data from the SDSS-III/APOGEE survey as released in the Data Release 12 (Alam et al. 2015). The APOGEE data set is rich and we expect that it will enable significant developments in Milky Way science. We have documented the methods by which APOGEE data can be accessed, and attempt to present information that will provide information about its reliability and limitations. 
Stellar parameters and abundances have been calibrated and compared with the literature using observations of stellar clusters and of stars with previously measured abundances. The internal precision of the APOGEE abundances is typcially 0.05-0.1 dex, judging from the internal scatter of abundances within clusters. External accuracy of the abundances is challenging to assess, but may be good only to $0.1-0.2$ dex. Stellar parameters and abundances for cooler stars $\left(T_{\text {eff }}<4000\right)$ may be more uncertain.

The automatic determination of stellar parameters and abundances presents a challenging problem, and we do not claim to be currently doing the most optimal job. Work is ongoing with a number of issues, including:

1. implementation of a correction for persistence in the SDSS-III/APOGEE data;

2. improvement of sky emission and telluric absorption correction;

3. improvement of radial velocities, especially for hotter stars and spectra with lower $\mathrm{S} / \mathrm{N}$;

4. continued revision of line lists;

5. incorporation of rotation where needed;

6. extension to cooler temps using MARCS model atmospheres and the Turbospectrum synthesis code (see Zamora et al. 2015);

7. investigation of individual element grids, rather than using the element family grids to solve for individual element abundances;

8. investigation of correlated errors;

9. improvments in handling of non-detections/upper limits;

10. investigation of issues with derivation of surface gravity.

Nevertheless, the DR12 results have proven to be useful and of a quality to conduct a wide variety of science.

As the study of Milky Way stars continues with SDSS-IV/ APOGEE-2, there will be future data releases, and we anticipate that at least some of these will include improved re-analysis of SDSS-III/APOGEE data.

Funding for SDSS-III has been provided by the Alfred P. Sloan Foundation, the Participating Institutions, the National Science Foundation, and the U.S. Department of Energy Office of Science. The SDSS-III web site is http://www.sdss3.org/.

SDSS-III is managed by the Astrophysical Research Consortium for the Participating Institutions of the SDSS-III Collaboration including the University of Arizona, the Brazilian Participation Group, Brookhaven National Laboratory, Carnegie Mellon University, University of Florida, the French Participation Group, the German Participation Group, Harvard University, the Instituto de Astrofisica de Canarias, the Michigan State/Notre Dame/JINA Participation Group, Johns Hopkins University, Lawrence Berkeley National Laboratory, Max Planck Institute for Astrophysics, Max Planck Institute for Extraterrestrial Physics, New Mexico State University, New York University, Ohio State University, Pennsylvania State University, University of Portsmouth, Princeton University, the Spanish Participation Group, University of Tokyo, University of Utah, Vanderbilt University, University of Virginia, University of Washington, and Yale University.

J. A. H., S. R. M., and V. V. S. acknowledge support for this research from the National Science Foundation (AST1109178). J. A. J. and M. P. also acknowledge support from the National Science Foundation (AST-1211673).
C. A. P. is thankful for support from the Spanish Ministry of Economy and Competitiveness (MINECO) through grant AYA2014-56359-P.

D. A. G. H. and O. Z. acknowledge support provided by the Spanish Ministry of Economy and Competitiveness under grant AYA-2011-27754.

T. C. B. acknowledges partial support for this work from grants PHY 08-22648; Physics Frontier Center/Joint Institute or Nuclear Astrophysics (JINA), and PHY 14-30152; Physics Frontier Center/JINA Center for the Evolution of the Elements (JINA-CEE), awarded by the US National Science Foundation. S. M. has been supported by the János Bolyai Research Scholarship of the Hungarian Academy of Sciences.

\section{REFERENCES}

Ahn, C. P., Alexandroff, R., Allende Prieto, C., et al. 2014, ApJS, 211, 17 Alam, S., Albareti, F. D., Allende Prieto, C., et al. 2015, arXiv:1501.00963 Allende Prieto, C., Beers, T. C., Wilhelm, R., et al. 2006, ApJ, 636, 804 Asplund, M., Grevesse, N., \& Sauval, A. J. 2005, in ASP Conf. Ser. 336, Cosmic Abundances as Records of Stellar Evolution and Nucleosynthesis, ed. T. G. Barnes, III \& F. N. Bash (San Francisco, CA: ASP), 25

Asplund, M., Grevesse, N., Sauval, A. J., \& Scott, P. 2009, ARA\&A, 47, 481 Barrado y Navascués, D., Deliyannis, C. P., \& Stauffer, J. R. 2001, ApJ, 549, 452 Bensby, T., Feltzing, S., \& Oey, M. S. 2014, A\&A, 562, A71

Bessell, M. S., Castelli, F., \& Plez, B. 1998, A\&A, 333, 231

Bovy, J., Nidever, D. L., Rix, H.-W., et al. 2014, ApJ, 790, 127

Bragaglia, A., Carretta, E., Gratton, R. G., et al. 2001, AJ, 121, 327

Bressan, A., Marigo, P., Girardi, L., et al. 2012, MNRAS, 427, 127

Carney, B. W., Gray, D. F., Yong, D., et al. 2008, AJ, 135, 892

Carraro, G., Bresolin, F., Villanova, S., et al. 2004, AJ, 128, 1676

Carraro, G., Villanova, S., Demarque, P., et al. 2006, ApJ, 643, 1151

Carretta, E., Bragaglia, A., Gratton, R., D’Orazi, V., \& Lucatello, S. 2009, A\&A, 508, 695

Casagrande, L., Silva Aguirre, V., Stello, D., et al. 2014, ApJ, 787, 110

Castelli, F., \& Kurucz, R. L. 2004, arXiv:astro-ph/0405087

Cunha, K., Smith, V. V., Johnson, J. A., et al. 2015, ApJL, 798, L41

Eisenstein, D. J., Weinberg, D. H., Agol, E., et al. 2011, AJ, 142, 72

González Hernández, J. I., \& Bonifacio, P. 2009, A\&A, 497, 497

Gunn, J. E., Siegmund, W. A., Mannery, E. J., et al. 2006, AJ, 131, 2332

Hekker, S., Elsworth, Y., Mosser, B., et al. 2013, A\&A, 556, A59

Hinkle, K., Wallace, L., \& Livingston, W. 1995, PASP, 107, 1042

Jacobson, H. R., Friel, E. D., \& Pilachowski, C. A. 2011a, AJ, 141, 58

Jacobson, H. R., Pilachowski, C. A., \& Friel, E. D. 2011b, AJ, 142, 59

Koesterke, L. 2009, in AIP Conf. Ser. 1171, ed. I. Hubeny et al. (Melville, NY: AIP), 73

Kurucz, R. L. 1979, ApJS, 40, 1

Lamb, M. P., Venn, K. A., Shetrone, M. D., Sakari, C. M., \& Pritzl, B. J. 2015, MNRAS, 448, 42

Livingston, W., \& Wallace, L. 1991, An Atlas of the Solar Spectrum in the Infrared from 1850 to $9000 \mathrm{~cm}-1$ (1.1 to 5.4 micrometer) (Tucson, AZ: National Solar Observatory)

Majewski, S. R., Zasowski, G., \& Nidever, D. L. 2011, ApJ, 739, 25

Mészáros, S., Allende Prieto, C., Edvardsson, B., et al. 2012, AJ, 144, 120

Mészáros, S., Holtzman, J., García Pérez, A. E., et al. 2013, AJ, 146, 133

Mészáros, S., Martell, S. L., Shetrone, M., et al. 2015, AJ, 149, 153

Nidever, D. L., Holtzman, J. A., Allende Prieto, C., et al. 2015, arXiv: 1501.03742

Pinsonneault, M. H., Elsworth, Y., Epstein, C., et al. 2014, ApJS, 215, 19

Ramírez, I., \& Allende Prieto, C. 2011, ApJ, 743, 135

Reddy, B. E., Lambert, D. L., \& Allende Prieto, C. 2006, MNRAS, 367, 1329

Reddy, B. E., Tomkin, J., Lambert, D. L., \& Allende Prieto, C. 2003, MNRAS, 340, 304

Seaman, R., Pence, W., \& White, R. 2010, ascl soft, ascl:1010.002

Smith, V. V., Cunha, K., Shetrone, M. D., et al. 2013, ApJ, 765, 16

Sneden, C., Lucatello, S., Ram, R. S., Brooke, J. S. A., \& Bernath, P. 2014, ApJS, 214, 26

Soderblom, D. R., Laskar, T., Valenti, J. A., Stauffer, J. R., \& Rebull, L. M. 2009, AJ, 138, 1292

Stello, D., Huber, D., Bedding, T. R., et al. 2013, ApJL, 765, L41

Zamora, O., Garcia-Hernandez, D. A., Allende Prieto, C., et al. 2015, arXiv: 1502.05237

Zasowski, G., Johnson, J. A., Frinchaboy, P. M., et al. 2013, AJ, 146, 81 